TRANSACTIONS OF THE

AMERICAN MATHEMATICAL SOCIETY

Volume 358, Number 7, Pages 3239-3262

S 0002-9947(06)03899-2

Article electronically published on February 20, 2006

\title{
AN INFINITARY EXTENSION OF THE GRAHAM-ROTHSCHILD PARAMETER SETS THEOREM
}

\author{
TIMOTHY J. CARLSON, NEIL HINDMAN, AND DONA STRAUSS
}

\begin{abstract}
The Graham-Rothschild Parameter Sets Theorem is one of the most powerful results of Ramsey Theory. (The Hales-Jewett Theorem is its most trivial instance.) Using the algebra of $\beta S$, the Stone-Čech compactification of a discrete semigroup, we derive an infinitary extension of the GrahamRothschild Parameter Sets Theorem. Even the simplest finite instance of this extension is a significant extension of the original. The original theorem says that whenever $k<m$ in $\mathbb{N}$ and the $k$-parameter words are colored with finitely many colors, there exist a color and an $m$-parameter word $w$ with the property that whenever a $k$-parameter word of length $m$ is substituted in $w$, the result is in the specified color. The "simplest finite instance" referred to above is that, given finite colorings of the $k$-parameter words for each $k<m$, there is one $m$-parameter word which works for each $k$. Some additional Ramsey Theoretic consequences are derived.

We also observe that, unlike any other Ramsey Theoretic result of which we are aware, central sets are not necessarily good enough for even the $k=1$ and $m=2$ version of the Graham-Rothschild Parameter Sets Theorem.
\end{abstract}

\section{INTRODUCTION}

Throught this paper $A$ will denote a nonempty set and $D$ will denote a set with a binary operation mapping $(f, g) \in D \times D$ to $f g \in D$. We assume that $D$ has a nonempty set $E$ of right identities for this operation. We also assume that, for each $f \in D$, we have defined a mapping $T_{f}: A \rightarrow A$. We shall call $\left(A, D, E,\left\langle T_{f}\right\rangle_{f \in D}\right)$ a parameter system.

We write $\omega$ for the set $\{0,1,2, \ldots\}$ of finite ordinals and $\mathbb{N}=\omega \backslash\{0\}$. We choose a set $V=\left\{\nu_{n}: n \in \omega\right\}$ such that $A \cap(D \times V)=\emptyset$ and define $W$ to be the semigroup of words over the alphabet $A \cup(D \times V)$, with concatenation as the semigroup operation. (Formally a word $w$ is a function from an initial segment $\{0,1, \ldots, k-1\}$ of $\omega$ to the alphabet, and the length $\ell(w)$ of $w$ is $k$. We shall occasionally need to resort to this formal meaning, so that if $i \in\{0,1, \ldots, \ell(w)-1\}$, then $w(i)$ denotes the $(i+1)$ st letter of $w$.)

For each $n \in \mathbb{N}$, we define $W_{n}$ to be the set of words over the alphabet $A \cup$ $\left(D \times\left\{\nu_{0}, \nu_{1}, \ldots, \nu_{n-1}\right\}\right)$, and we define $W_{0}$ to be the set of words over $A$. We note that each $W_{n}$ is a subsemigroup of $W$.

Received by the editors February 20, 2004 and, in revised form, September 14, 2004.

2000 Mathematics Subject Classification. Primary 05D10.

The second author acknowledges support received from the National Science Foundation (USA) via grant DMS-0070593. 
Definition 1.1. Let $n \in \mathbb{N}$ and $k \in \omega$ with $k \leq n$. Then $S\left(\begin{array}{l}n \\ k\end{array}\right)$ is the set of all words $w \in W_{k}$ of length $n$ such that

(1) for each $i \in\{0,1, \ldots, k-1\}$, if any, some member of $E \times\left\{\nu_{i}\right\}$ occurs in $w$;

(2) for each $i \in\{0,1, \ldots, k-1\}$, if any, the first occurrence of a member $\left(s, \nu_{i}\right)$ of $D \times\left\{\nu_{i}\right\}$ has $s \in E$;

(3) for each $i \in\{0,1, \ldots, k-2\}$, if any, the first occurrence of a member of $D \times\left\{\nu_{i}\right\}$ in $w$ precedes the first occurrence of a member of $D \times\left\{\nu_{i+1}\right\}$.

Definition 1.2. Let $k \in \mathbb{N}$. Then the set of $k$-parameter words is $S_{k}=\bigcup_{n=k}^{\infty} S\left(\begin{array}{l}n \\ k\end{array}\right)$.

Of course, $S\left(\begin{array}{l}n \\ k\end{array}\right)$ and $S_{k}$ depend on $A, D$ and $E$, as well as $n$ and $k$. Since we consider only one parameter system at a time throughout most of the paper, we shall not normally indicate this dependence in the notation. In a context where more than one parameter system is used, we shall use $S\left(\begin{array}{l}n \\ k\end{array}\right)(\Gamma)$ and $S_{k}(\Gamma)$ for the sets defined above by the parameter system $\Gamma$. If $k=0$, when we write $S_{k}$ we simply mean $W_{0}$.

For each $i \in \omega$, we choose a member $v_{i}$ of $E \times\left\{\nu_{i}\right\}$. If $D=\{e\}$ and $T_{e}: A \rightarrow A$ is the identity, then the $k$-parameter words are known as the $k$-variable words, where each $v_{i}$ is a "variable".

Given $w \in S_{n}$ and $u \in W$ with $\ell(u)=n$, we define $w\langle u\rangle$ to be the word with length $\ell(w)$ such that for $i \in\{0,1, \ldots, \ell(w)-1\}$

$$
w\langle u\rangle(i)=\left\{\begin{array}{cl}
w(i) & \text { if } w(i) \in A, \\
T_{s}(u(j)) & \text { if } w(i)=\left(s, \nu_{j}\right) \text { and } u(j) \in A, \\
\left(s t, \nu_{l}\right) & \text { if } w(i)=\left(s, \nu_{j}\right) \text { and } u(j)=\left(t, \nu_{l}\right) .
\end{array}\right.
$$

For example, suppose that $A=\{a, b, c\}$ and $D=\{e, f, g\}$ is a group, with $e$

$$
a \mapsto b
$$

the identity and $g=f^{2}$. Suppose also that $T_{f}: \quad b \mapsto c, T_{g}=T_{f}{ }^{2}$, and $T_{e}$ is the

$$
c \mapsto a
$$

identity. If $w=v_{0} a v_{1}\left(f, \nu_{1}\right) b\left(g, \nu_{0}\right)=\left(e, \nu_{0}\right) a\left(e, \nu_{1}\right)\left(f, \nu_{1}\right) b\left(g, \nu_{0}\right)$, and $u=\left(f, \nu_{2}\right) a$, then $w\langle u\rangle=\left(e f, \nu_{2}\right) a T_{e}(a) T_{f}(a) b\left(g f, \nu_{2}\right)=\left(f, \nu_{2}\right) a a b b\left(e, \nu_{2}\right)$.

If $D=\{e\}$ and $T_{e}$ is the identity map, then $w\langle u\rangle$ is simply the result of replacing each occurrence of $v_{i}$ in $w$ by $u(i)$.

The following theorem is the Graham-Rothschild Parameter Sets Theorem. We use the standard "chromatic" terminology for Ramsey Theoretic results. When we say that a set is finitely colored, we mean that there is a function from that set to a finite set. A set is monochrome provided the given coloring function is constant on it. If $D$ is a group, then the statement that $\left\langle T_{f}\right\rangle_{f \in D}$ is an action of $D$ on $A$ is the assertion that $T_{f} \circ T_{g}=T_{f g}$ for all $f, g \in D$ and that $T_{e}$ is the identity map.

Theorem 1.3 (Graham-Rothschild). Assume that the alphabet $A$ is finite, that $D$ is a finite group, and that $\left\langle T_{f}\right\rangle_{f \in D}$ is an action of $D$ on $A$. Let $m, k \in \omega$ with $m>k$ and let $S_{k}$ be finitely colored. There exists $w \in S_{m}$ such that $\left\{w\langle u\rangle: u \in S\left(\begin{array}{c}m \\ k\end{array}\right)\right\}$ is monochrome.

Proof. 6], or see [14] for a shorter proof. 
The case $m=1, k=0$, and $D=\{e\}$ of Theorem 1.3 is the Hales-Jewett Theorem [8]. The version of Theorem 1.3 which has $D=\{e\}$ is commonly cited in the literature as the Graham-Rothschild Parameter Sets Theorem, and most of the standard consequences of the Graham-Rothschild Theorem are consequences of this special case. We shall show in Theorem 5.1 that Theorem 1.3 is derivable from this special case. This is true even if the assumptions of Theorem 1.3 are significantly weakened.

The restriction on the order of first appearances of members of $D \times\left\{\nu_{i}\right\}$ in the definition of an $n$-parameter word may seem unnatural. Note however, that without that restriction, the $m=3, k=2$, and $D=\{e\}$ case of Theorem 1.3 is false. (Simply color the two variable words according to whether the first occurrence of $v_{0}$ precedes or follows the first occurrence of $v_{1}$.)

The Graham-Rothschild Parameter Sets Theorem has been recognized for its power from the time of its appearance. Section 9 of [6] contains 13 corollaries. Included among these are four results that were known at the time (namely the Hales-Jewett Theorem, van der Waerden's Theorem, Ramsey's Theorem, and the finite version of the Finite Sums Theorem). We believe that the other nine were new at the time. (These include the finite version of the Finite Unions Theorem. While the infinite version of the Finite Unions Theorem is obviously derivable from the infinite version of the Finite Sums Theorem, the finite version of the Finite Unions Theorem is not obviously derivable from the finite version of the Finite Sums Theorem.) In introducing their article about the Graham-Rothschild Parameter Sets Theorem, Prömel and Voigt [14] wrote:

This is a complete analogue to Ramsey's theorem carried over to the structures of parameter sets and, as it turns out, Ramsey's theorem itself is an immediate consequence of the Graham-Rothschild theorem. But the concept of parameter sets does not only glue arithmetic progressions and finite sets together. Also, it provides a natural framework for seemingly different structures like Boolean lattices, partition lattices, hypergraphs and Deuber's $(m, p, c)$-sets, just to mention a few. So, the Graham-Rothschild theorem can be viewed as a starting point of Ramsey Theory.

Other strong consequences of infinitary results such as those established here are analogues of the Paris-Harrington Theorem [13]: the statement

(*) For any positive integers $c$ and $e$ there is a positive integer $N$ such that if $[N]^{e}$ is colored with $c$ colors there is a large homogeneous set of size at least $e+1$ (a set of integers is large if its cardinality is at least as large as its least element).

is true and cannot be proved in the formal theory of Peano Arithmetic. The statement $(\star)$ can be proved from the infinite version of Ramsey's Theorem by the same kind of compactness argument used to derive the finite version of Ramsey's Theorem. Perhaps the most important feature of the Paris-Harrington Theorem is that it was the first example of a striking combinatorial fact which cannot be proved in the theory of formal Peano Arithmetic. Gödel's incompleteness theorem showed that there are finitary truths not provable from Peano Arithmetic, but the examples given before the Paris-Harrington Theorem were not very satisfying mathematically. By similar sorts of compactness arguments, principles similar to $(\star)$ can be derived 
from infinitary theorems on variable words. For example, Theorem 5.4 implies the following principle:

$(\star \star)$ For any finite alphabet $A$ and any positive integers $c$ and $e$ there is a positive integer $N$ such that whenever the $e$-variable words of length $N$ are colored with $c$ colors there is an integer $m \geq e+1$ and a large $m$-variable word of length $N$ all of whose $e$-variable reductions have the same color (an $m$ variable word is large if $k \leq m$ where the first occurence of a variable is in position $k$ ).

While we do not have tight bounds on what is required to prove $(\star \star)$, it cannot be proved in Peano Arithmetic since it easily implies $(\star)$. (Given a coloring of $[N]^{e}$, color an $e$-variable word of length $N$ according to the color of the set of those $k$ such that the first occurence of some variable occurs in position $k$.)

The following extension of the $D=\{e\}$ version of Theorem 1.3 is not new, being a direct consequence of [2, Theorem 10]. However, it is certainly not well known, even among the experts, and we shall present its derivation in Section 5. Given a set $B$, we write $\mathcal{P}_{f}(B)$ for the set of finite nonempty subsets of $B$.

Theorem 1.4. Assume that $A$ is finite, $D=\{e\}$, and, for each $n \in \omega, S_{n}$ has been finitely colored. Then, there exists a sequence $\left\langle w_{n}\right\rangle_{n<\omega}$ with each $w_{n} \in S_{n}$ such that for every $m \in \omega$,

$$
S_{m} \cap\left\{\prod_{n \in F} w_{n}\left\langle u_{n}\right\rangle: F \in \mathcal{P}_{f}(\omega) \text { and for all } n \in F, u_{n} \in \bigcup_{i=0}^{\min F} S\left(\begin{array}{c}
n \\
i
\end{array}\right)\right\}
$$

is monochrome. (That is, the color of $\prod_{n \in F} w_{n}\left\langle u_{n}\right\rangle$ is determined solely by the number of variables in $\prod_{n \in F} w_{n}\left\langle u_{n}\right\rangle$.)

In Section 3 we shall derive the following extension of Theorems 1.3 and 1.4 .

Corollary 1.5. Assume that, for each $n \in \omega, S_{n}$ has been finitely colored and that, for each $n \in \omega$ and each $i \in\{0,1, \ldots, n\}, H_{n, i}$ is a finite subset of $S\left(\begin{array}{c}n \\ i\end{array}\right)$. Then, there exists a sequence $\left\langle w_{n}\right\rangle_{n<\omega}$ with each $w_{n} \in S_{n}$ such that for every $m \in \omega$,

$$
S_{m} \cap\left\{\prod_{n \in F} w_{n}\left\langle u_{n}\right\rangle: F \in \mathcal{P}_{f}(\omega) \text { and for all } n \in F, u_{n} \in \bigcup_{i=0}^{\min F} H_{n, i}\right\}
$$

is monochrome. (That is, the color of $\prod_{n \in F} w_{n}\left\langle u_{n}\right\rangle$ is determined solely by the number of parameters in $\prod_{n \in F} w_{n}\left\langle u_{n}\right\rangle$.)

The special case $D=\{e\}$ of Corollary [1.5 can be derived from [2, Theorem 15] by an argument similar to the one we shall use to establish Theorem 1.4

Note that if $A$ and $D$ are finite, one may take $H_{n, i}=S\left(\begin{array}{c}n \\ i\end{array}\right)$ in Corollary 1.5.

Perhaps somewhat easier to absorb is the following corollary to Corollary 1.5.

Corollary 1.6. Let $m \in \mathbb{N}$. Suppose that we have a finite coloring of $\bigcup_{i=0}^{m} S_{i}$ and a finite subset $H_{i}$ of $S\left(\begin{array}{c}m \\ i\end{array}\right)$ for each $i \in\{0,1, \ldots, m\}$. Then there exists a sequence $\left\langle w_{n}\right\rangle_{n=0}^{\infty}$ in $S_{m}$ such that for every $l \in\{0,1, \ldots, m\}$,

$$
S_{l} \cap\left\{\prod_{n \in F} w_{n}\left\langle u_{n}\right\rangle: F \in \mathcal{P}_{f}(\omega) \text { and for all } n \in F, u_{n} \in \bigcup_{i=0}^{m} H_{i}\right\}
$$

is monochrome. 
In particular, one immediately has the following extension of Theorem 1.3. (In Section 3 we shall describe how this extension can be derived from Theorem 1.3 without using the results of Section 2.)

Corollary 1.7. Assume that the alphabet $A$ is finite, that $D$ is a finite group, and that $\left\langle T_{f}\right\rangle_{f \in D}$ is an action of $D$ on $A$. Let $m, k \in \omega$ with $m>k$ and let $S_{k}$ and $S_{m}$ be finitely colored. There exists a sequence $\left\langle w_{t}\right\rangle_{t=0}^{\infty}$ in $S_{m}$ such that $\left\{\prod_{t \in F} w_{t}: F \in \mathcal{P}_{f}(\omega)\right\}$ is monochrome and $\left\{\prod_{t \in F} w_{t}\left\langle u_{t}\right\rangle: F \in \mathcal{P}_{f}(\omega)\right.$ and for each $\left.n \in F, u_{n} \in S\left(\begin{array}{c}m \\ k\end{array}\right)\right\}$ is monochrome.

We derive Corollary 1.5 as a straightforward consequence of the existence of a particular chain of idempotents $\left\langle p_{n}\right\rangle_{n=0}^{\infty}$, where each $p_{n} \in \beta S_{n}$. Section 2 is devoted to the proof of the existence of this special chain of idempotents. (More precisely, Corollary 1.5 is a special case of Theorem 3.2, the statement of which requires the introduction of additional terminology.)

Let us briefly review some facts about the Stone-Čech compactification $\beta T$ of a (discrete) semigroup $(T, \cdot)$. We take the points of $\beta T$ to be the ultrafilters on $T$, the principal ultrafilters being identified with the points of $T$. Given a set $A \subseteq T$, $\bar{A}=\{p \in \beta T: A \in p\}$. The set $\{\bar{A}: A \subseteq T\}$ is a basis for the open sets (as well as a basis for the closed sets) of $\beta T$. If $R \subseteq T$ we shall identify an ultrafilter $p$ on $R$ with the ultrafilter $\{A \subseteq T: A \cap R \in p\}$ and thereby pretend that $\beta R \subseteq \beta T$.

There is a natural extension of the operation - of $T$ to $\beta T$ making $\beta T$ a compact right topological semigroup with $T$ contained in its topological center. This says that for each $p \in \beta T$ the function $\rho_{p}: \beta T \rightarrow \beta T$ is continuous and for each $x \in T$, the function $\lambda_{x}: \beta T \rightarrow \beta T$ is continuous, where $\rho_{p}(q)=q \cdot p$ and $\lambda_{x}(q)=x \cdot q$. Given $B \subseteq T$ and $x \in T$, let $x^{-1} B=\{y \in T: x \cdot y \in B\}$. Then for any $p, q \in \beta T$ and any $B \subseteq T$, one has that $B \in p \cdot q$ if and only if $\left\{x \in T: x^{-1} B \in q\right\} \in p$. See [10, for an elementary introduction to the semigroup $\beta T$ and for any unfamiliar algebraic facts encountered in this paper.

A subset $V$ of a semigroup $T$ is called a left ideal if it is nonempty and $T V \subseteq V$. It is called a right ideal if it is nonempty and $V T \subseteq V$. It is called a two-sided ideal, or simply an ideal, if it is both a left ideal and a right ideal. Any compact Hausdorff right topological semigroup $T$ has a smallest two-sided ideal $K(T)$ which is the union of all of the minimal left ideals of $T$ and is also the union of all of the minimal right ideals of $T$. If $x \in K(T)$, then $x T$ is the minimal right ideal with $x$ as a member, and $T x$ is the minimal left ideal with $x$ as a member. The intersection of any minimal left ideal and any minimal right ideal is a group. In particular there are idempotents in the smallest ideal. There is a partial ordering of the idempotents of $T$ determined by $p \leq q$ if and only if $p=p \cdot q=q \cdot p$. An idempotent $p$ is minimal with respect to this order if and only if $p \in K(T)$ [10, Theorem 1.59]. Such an idempotent is called simply "minimal".

A subset $B$ of a discrete semigroup $T$ is central if and only if it is a member of a minimal idempotent of $\beta T$. Central sets are known to have remarkably strong combinatorial properties. For example [5. Theorem 8.22] any central subset of $\mathbb{N}$ contains solutions to all partition regular systems of homogeneous linear equations. See [10] and 9] for numerous other combinatorial conclusions about central sets.

Loosely speaking, Theorem 3.2 says that when one is constructing the sequence $\left\langle w_{n}\right\rangle_{n=0}^{\infty}$ in the statement of Corollary 1.5, one can take $w_{n}$ to be any member of some central subset of $S_{n}$. 
It was shown in the proof of [1, Theorem 4.1] that the $k=0, m=1$, and $D=\{e\}$ case of Corollary 1.7 (and in particular the Hales-Jewett Theorem) holds, where $\varphi^{-1}[\{i\}]$ is any central subset of $W_{0}$. In fact all previous Ramsey Theoretic results of which we are aware that could be stated in terms of a finite partition of a semigroup had a conclusion valid for arbitrary central sets. We shall show at the end of Section 3 that there is a central subset $B$ of $S_{1}$ for which the conclusion of Theorem 1.3 fails with $m=2$ and $D=\{e\}$. (That is, there is no $w \in S_{2}$ such that $w\langle u\rangle \in B$ for every $u \in S\left(\begin{array}{l}2 \\ 1\end{array}\right)$.)

In Section 4 we shall derive some Ramsey Theoretic consequences of the results of Section 3. Additional consequences will appear in [3].

\section{A ChAin OF IDEMPotents}

Recall that we are assuming that we have a nonempty alphabet $A$, a set $D$ with a binary operation, a nonempty set $E$ of right identities for $D$, and a mapping $T_{f}: A \rightarrow A$ defined for every $f \in D$. Recall also that we have chosen a member $v_{i}$ of $E \times\left\{\nu_{i}\right\}$ for each $i \in \omega$.

Suppose that $u \in W$ has length $n$. We shall define a homomorphism $h_{u}: W \rightarrow$ $W$ by first defining $h_{u}$ on all elements of $A \cup(D \times V)$. So let $w \in A \cup(D \times V)$. Then

$$
h_{u}(w)=\left\{\begin{array}{cl}
w & \text { if } w \in A \\
T_{s}(u(j)) & \text { if } w=\left(s, \nu_{j}\right), j<n, \text { and } u(j) \in A \\
\left(s t, \nu_{l}\right) & \text { if } w=\left(s, \nu_{j}\right), j<n, \text { and } u(j)=\left(t, \nu_{l}\right), \\
w & \text { if } w=\left(s, \nu_{j}\right) \text { and } j \geq n
\end{array}\right.
$$

Since $W$ is the free semigroup on $A \cup(D \times V), h_{u}$ extends to a unique homomorphism defined on $W$, which we also denote by $h_{u}$. Thus, if $w \in W$ and $\ell(w)=k$ one has that $\ell\left(h_{u}(w)\right)=k$, and for $i \in\{0,1, \ldots, k-1\}$,

$$
h_{u}(w)(i)=\left\{\begin{array}{cl}
w(i) & \text { if } w(i) \in A, \\
T_{s}(u(j)) & \text { if } w(i)=\left(s, \nu_{j}\right), j<n, \text { and } u(j) \in A, \\
\left(s t, \nu_{l}\right) & \text { if } w(i)=\left(s, \nu_{j}\right), j<n, \text { and } u(j)=\left(t, \nu_{l}\right), \\
w(i) & \text { if } w(i)=\left(s, \nu_{j}\right) \text { and } j \geq n
\end{array}\right.
$$

Observe that, if $n \in \mathbb{N}, w \in S_{n}, u \in W$, and $\ell(u)=n$, then $h_{u}(w)=w\langle u\rangle$. Observe also that if $u \in S\left(\begin{array}{l}n \\ k\end{array}\right)$, then $h_{u}: W_{n} \rightarrow W_{k}$ and $h_{u}: S_{n} \rightarrow S_{k}$.

We shall also use $h_{u}$ to denote the continuous extension of $h_{u}$ from $\beta W$ to itself.

Lemma 2.1. Let $u \in W$. Then $h_{u}$ is a homomorphism from $\beta W$ to $\beta W$.

Proof. This is [10, Corollary 4.22] due originally to P. Milnes in [12].

In the following definition, and throughout the rest of this paper, when we write an expression such as $v_{i} \cdots v_{j}$, we assume that all intervening values of the subscript occur in order.

Definition 2.2. Let $n \in \mathbb{N}$ with $n \geq 2$.

(a) For $i \in\{0,1, \ldots, n-1\}, w_{n, i}$ is the word obtained from $v_{0} \cdots v_{n-1}$ by deleting $v_{i}$. 
(b) For $i \in\{0,1, \ldots, n-1\}$,

$$
\begin{aligned}
U_{n, i}=\{w \in W: \quad & \ell(w)=n, w(i) \in A \cup\left\{\left(s, \nu_{l}\right): s \in D \text { and } 0 \leq l<i\right\}, \\
& \text { and for all } j \in\{0,1, \ldots, n-1\}, \text { if } j<i, \text { then } \\
& \left.w(j) \in E \times\left\{\nu_{j}\right\} \text { and if } j>i, \text { then } w(j) \in E \times\left\{\nu_{j-1}\right\}\right\} .
\end{aligned}
$$

Thus, if $0<i<n-1$, a member of $U_{n, i}$ is of the form $w_{0} \cdots w_{i-1} t w_{i} \cdots w_{n-2}$ where $t \in A \cup\left(D \times\left\{\nu_{0}, \nu_{1}, \ldots, \nu_{i-1}\right\}\right)$ and each $w_{i} \in E \times\left\{\nu_{i}\right\}$.

Note that for any $n \in \mathbb{N}$ with $n \geq 2, S\left(\begin{array}{c}n \\ n-1\end{array}\right)=\bigcup_{i=0}^{n-1} U_{n, i}$.

Lemma 2.3. Let $n \in \mathbb{N}$. Then $h_{v_{0} \cdots v_{n-1}}$ is the identity on $W_{n}$.

Proof. Since $h_{v_{0} \cdots v_{n-1}}$ is a homomorphism, it suffices to show that $h_{v_{0} \cdots v_{n-1}}(x)=x$ for any $x \in A \cup\left(D \times\left\{\nu_{0}, \nu_{1}, \ldots, \nu_{n-1}\right\}\right)$. If $x \in A$, this is immediate. If $x=\left(s, \nu_{i}\right)$, then $h_{v_{0} \cdots v_{n-1}}(x)=\left(s e, \nu_{i}\right)$ for some $e \in E$ and $s e=s$ because $e$ is a right identity for $D$.

Lemma 2.4. Let $n \in \mathbb{N}$ with $n \geq 2$.

(a) If $i \in\{0,1, \ldots, n-1\}$, and $u \in U_{n, i}$, then $h_{u} \circ h_{w_{n, i}}$ is equal to the identity on $W_{n-1}$.

(b) If $i, k \in\{0,1, \ldots, n-1\}, i<k$, and $u \in U_{n+1, i}$, then for all $x \in W_{n}$, $h_{u}\left(h_{w_{n+1, k}}(x)\right)=h_{w_{n, k-1}}\left(h_{u}(x)\right)$.

Proof. (a) Since $h_{u} \circ h_{w_{n, i}}$ is a homomorphism, it suffices to let $x \in A \cup$ $\left(D \times\left\{\nu_{0}, \nu_{1}, \ldots, \nu_{n-2}\right\}\right)$ and show that $h_{u} \circ h_{w_{n, i}}(x)=x$. If $x \in A$, this is immediate. So assume that $x=\left(s, \nu_{j}\right)$ for some $j \in\{0,1, \ldots, n-2\}$. If $j<i$, then $w_{n, i}(j)=v_{j}$ and $u(j) \in E \times\left\{\nu_{j}\right\}$, so $h_{u} \circ h_{w_{n, i}}(x)=x$. If $j \geq i$, then $w_{n, i}(j)=v_{j+1}$ and $u(j+1) \in E \times\left\{\nu_{j}\right\}$, and so $h_{u} \circ h_{w_{n, i}}(x)=x$.

(b) Since $h_{u} \circ h_{w_{n+1, k}}$ and $h_{w_{n, k-1}} \circ h_{u}$ are homomorphisms, it suffices to establish the conclusion for $x \in A \cup\left(D \times\left\{\nu_{0}, \nu_{1}, \ldots, \nu_{n-1}\right\}\right)$. The case in which $x \in A$ is trivial. So assume that $x=\left(s, \nu_{j}\right)$ for some $j \in\{0,1, \ldots, n-1\}$.

Case 1. $j<i$. Then $w_{n+1, k}(j)=w_{n, k-1}(j)=v_{j}$ and $u(j)=\left(e, \nu_{j}\right)$ for some $e \in E$. Therefore, $h_{w_{n+1, k}}(x)=h_{u}(x)=h_{w_{n, k-1}}(x)=\left(s e, \nu_{j}\right)=x$. Consequently $h_{u}\left(h_{w_{n+1, k}}(x)\right)=h_{u}(x)=x$ and $h_{w_{n, k-1}}\left(h_{u}(x)\right)=h_{w_{n, k-1}}(x)=x$.

Case 2. $j=i$. Then $w_{n+1, k}(j)=v_{j}$, so $h_{w_{n+1, k}}(x)=\left(s, \nu_{j}\right)$.

Case 2a. $u(j) \in A$. Then $h_{u}\left(h_{w_{n+1, k}}(x)\right)=T_{s}(u(j))$. Also, $h_{u}(x)=T_{s}(u(j)) \in A$, and so $h_{w_{n, k-1}}\left(h_{u}(x)\right)=T_{s}(u(j))$.

Case 2b. $u(j)=\left(t, \nu_{l}\right)$ for some $t \in D$ and some $l<i$. Then $h_{u}\left(h_{w_{n+1, k}}(x)\right)=\left(s t, \nu_{l}\right)$. Also, $h_{u}(x)=\left(s t, \nu_{l}\right)$ and $w_{n, k-1}(l)=v_{l}$ so $h_{w_{n, k-1}}\left(h_{u}(x)\right)=\left(s t, \nu_{l}\right)$.

Case 3. $i<j<k$. Then $w_{n+1, k}(j)=v_{j}, u(j)=\left(e, \nu_{j-1}\right)$ for some $e \in E$, and $w_{n, k-1}(j-1)=v_{j-1}$. Therefore $h_{w_{n+1, k}}(x)=\left(s, \nu_{j}\right)$, so $h_{u}\left(h_{w_{n+1, k}}(x)\right)=\left(s, \nu_{j-1}\right)$. Also, $h_{u}(x)=\left(s, \nu_{j-1}\right)$, so $h_{w_{n, k-1}}\left(h_{u}(x)\right)=\left(s, \nu_{j-1}\right)$.

Case 4. $k \leq j$. Then $w_{n+1, k}(j)=v_{j+1}, u(j)=\left(e, \nu_{j-1}\right)$ and $u(j+1)=\left(f, \nu_{j}\right)$ for some $e, f \in E$, and $w_{n, k-1}(j-1)=v_{j}$. Therefore $h_{w_{n+1, k}}(x)=\left(s, \nu_{j+1}\right)$ so $h_{u}\left(h_{w_{n+1, k}}(x)\right)=\left(s, \nu_{j}\right)$. Also, $h_{u}(x)=\left(s, \nu_{j-1}\right)$, and so $h_{w_{n, k-1}}\left(h_{u}(x)\right)=$ $\left(s, \nu_{j}\right)$.

It is standard to define partial orders of idempotents of a semigroup $T$ by $p \leq_{R} q$ if and only if $p \in q T$ and $p \leq_{L} q$ if and only if $p \in T q$. We observe that these are 
equivalent respectively to $p=q p$ and $p=p q$. (If $p=q x$, then $q p=q q x=q x=p$.) We extend these definitions to all of $\beta W$.

Definition 2.5. Let $p, q \in \beta W$. Then $p \leq_{L} q$ if and only if $p \in \beta W q$ and $p \leq_{R} q$ if and only if $p \in q \beta W$.

We shall use the obvious fact that, for any homomorphism $h: \beta W \rightarrow \beta W$, $p \leq_{L} q$ implies that $h(p) \leq_{L} h(q)$, and $p \leq_{R} q$ implies that $h(p) \leq_{R} h(q)$. We observe that, if $p$ and $q$ are idempotent, then $p \leq q$ if and only if $p \leq_{R} q$ and $p \leq_{L} q$.

Note that $\leq_{L}$ and $\leq_{R}$ are transitive but are not, in general, reflexive on $\beta W$.

We now state several simple algebraic facts which will be needed in the proof of Theorem 2.12

Lemma 2.6. Let $n \in \omega$.

(a) If $p \in \beta W_{n}, r \in \beta W$, and $p \leq_{R} r$, then $p=r y$ for some $y \in \beta W_{n}$.

(b) If $p \in \beta W_{n}, r \in \beta W$, and $p \leq_{L} r$, then $p=y r$ for some $y \in \beta W_{n}$.

(c) If $p, q \in \beta W_{n}, r \in K\left(\beta W_{n}\right), p \leq_{R} r$, and $q \leq_{R} r$, then $p \leq_{R} q$.

(d) If $p, q \in \beta W_{n}, r \in K\left(\beta W_{n}\right), p \leq_{L} r$, and $q \leq_{L} r$, then $p \leq_{L} q$.

Proof. We establish (a) and (c). For (a), we have that $p=r y$ for some $y \in \beta W$. Since $W \backslash W_{n}$ is an ideal of $W, \beta W \backslash \beta W_{n}$ is an ideal of $\beta W$ by [10, Corollary 4.18] and so $y \in \beta W_{n}$.

(c) Since $r \in K\left(\beta W_{n}\right)$, pick a minimal right ideal $R$ of $\beta W_{n}$ such that $r \in R$. Then by (a) $p \in R$ and similarly $q \in R$. Thus $p \in R=q \beta W_{n} \subseteq q \beta W$.

Lemma 2.7. Let $T$ be a compact right topological semigroup. If $L$ is a left ideal of $T$ and $R$ is a right ideal of $T$, then there is an idempotent $p \in R \cap L$ which is minimal in $T$.

Proof. By [10, Corollary 2.6 and Theorem 2.7] we may pick a minimal left ideal $L^{\prime} \subseteq L$ of $T$ and a minimal right ideal $R^{\prime} \subseteq R$ of $T$, and one has that $L^{\prime} \cap R^{\prime}$ is a group.

Definition 2.8. Let $n \in \mathbb{N}$. Then $Q_{n}=\left\{w \in W_{n}\right.$ : some member of $E \times\left\{\nu_{n-1}\right\}$ occurs in $w$ and occurs before any other member of $\left.D \times\left\{\nu_{n-1}\right\}\right\}$.

Lemma 2.9. Let $n \in \mathbb{N}$, let $p$ be an idempotent of $\beta S_{n}$ and let $q$ be a minimal idempotent of $\beta W_{n+1}$ such that $q \leq p$. If $Q_{n+1} \in q$, then $q$ is a minimal idempotent of $\beta S_{n+1}$.

Proof. Given $w \in S_{n}$ and $u \in Q_{n+1}, w u \in S_{n+1}$ and thus $S_{n+1} \in p q=q$. Therefore $q \in \beta S_{n+1}$, and is thus minimal with respect to $\leq$ in $\beta S_{n+1}$.

Lemma 2.10. Let $n \in \mathbb{N}$, let $p \in \beta W_{n}$, and let $r \in \beta W_{n+1}$. If $Q_{n+1} \in r$ and $q \in \operatorname{pr} \beta W_{n+1}$, then $Q_{n+1} \in q$.

Proof. We have that $W_{n} \in p$ and $Q_{n+1} \in r$, so $W_{n} Q_{n+1} W_{n+1} \in q$ and $W_{n} Q_{n+1} W_{n+1}$ $\subseteq Q_{n+1}$.

Lemma 2.11. Let $n \in \mathbb{N}$, let $p \in \beta W_{n-1}$, let $r \in \beta S_{n}$, and let $q \in K\left(\beta W_{n}\right)$. If $p q \leq_{R} r$, then $Q_{n} \in q$. 
Proof. Let $T=\left\{w \in W_{n}\right.$ : some member of $E \times\left\{\nu_{n-1}\right\}$ occurs in $\left.w\right\}$. Then $T$ is an ideal of $W_{n}$, so $\beta T$ is an ideal of $\beta W_{n}$, and therefore $K\left(\beta W_{n}\right) \subseteq \beta T$ and thus $T \in q$. Suppose that $T \backslash Q_{n} \in q$. We have that $p q=r x$ for some $x \in \beta W$. Also $W_{n-1}\left(T \backslash Q_{n}\right) \in p q$ and $S_{n} W \in r x$. This is a contradiction because $W_{n-1}\left(T \backslash Q_{n}\right) \cap S_{n} W=\emptyset$.

Theorem 10 of [2] and its consequence Theorem [5.4 follow from [2, Lemma 7.1] which establishes, in the case $D=\{e\}$ and $T_{e}$ is the identity on $A$, the existence of a sequence of idempotents $p_{n}$ in $\beta S_{n}$ for $n \in \omega$ such that for any $n \in \omega$ :

- $p_{n+1} \leq p_{n}$

- for any $m<n$ and each $u \in S\left(\begin{array}{c}m \\ n\end{array}\right), h_{u}\left(p_{n}\right)=p_{m}$.

Our main algebraic result, Theorem 2.12 below, shows that there is such a sequence for general $D$ and, moreover, one may choose each of the idempotents $p_{n}$ to be minimal in $\beta S_{n}$.

Recall that $W_{0}$ is the free semigroup (i.e., the set of words) on the alphabet $A$. Recall also that we do not need to assume that either $A$ or $D$ is finite for this result.

Theorem 2.12. Let $p$ be a minimal idempotent in $\beta W_{0}$. There is a sequence $\left\langle p_{n}\right\rangle_{n=0}^{\infty}$ such that:

(1) $p_{0}=p$;

(2) for each $n \in \mathbb{N}, p_{n}$ is a minimal idempotent of $\beta S_{n}$;

(3) for each $n \in \mathbb{N}, p_{n} \leq p_{n-1}$;

(4) for each $n \in \mathbb{N}$ and each $u \in S\left(\begin{array}{c}n \\ n-1\end{array}\right), h_{u}\left(p_{n}\right)=p_{n-1}$.

Further, $p_{1}$ can be any minimal idempotent of $\beta S_{1}$ such that $p_{1} \leq p_{0}$.

Proof. We first show how $p_{0}, p_{1}$ and $p_{2}$ can be defined. Let $p_{0}=p$ and let $p_{1}$ be any minimal idempotent of $\beta S_{1}$ such that $p_{1} \leq p_{0}$. Such exist because by Lemma 2.7 we may pick an idempotent

$$
p_{1} \in\left(p_{0} \cdot \beta S_{1}\right) \cap\left(\beta S_{1} \cdot p_{0}\right)
$$

which is minimal in $\beta S_{1}$. Then $p_{1} \leq p_{0}$. (We have $p_{1}=p_{0} x$ for some $x \in \beta S_{1}$, and so $p_{0} p_{1}=p_{0} p_{0} x=p_{0} x=p_{1}$. Similarly, $p_{1} p_{0}=p_{1}$.) Now $S\left(\begin{array}{l}1 \\ 0\end{array}\right)$ consists of all words of length 1 from the alphabet $A$. Thus if $u \in S\left(\begin{array}{l}1 \\ 0\end{array}\right)$, then $h_{u}\left[S_{1}\right] \subseteq W_{0}$ and $h_{u}$ is the identity on $W_{0}$. Therefore $h_{u}\left(p_{1}\right) \in \beta W_{0}$ and, since $h_{u}$ is a homomorphism, $h_{u}\left(p_{1}\right) \leq h_{u}\left(p_{0}\right)=p_{0}$. Since $p_{0}$ is minimal in $\beta W_{0}, h_{u}\left(p_{1}\right)=p_{0}$. (The argument in this paragraph is due to Andreas Blass, and first appeared in [1.)

Let $\alpha=h_{v_{1}}\left(p_{1}\right)$. Then $\alpha \in \beta W_{2}$, so by Lemma 2.7 we may pick an idempotent

$$
p_{2} \in\left(p_{1} \alpha \beta W_{2}\right) \cap\left(\beta W_{2} \alpha p_{1}\right)
$$

which is minimal in $\beta W_{2}$. Then, as in the previous paragraph, $p_{2} \leq p_{1}$. Since $S_{1} \in p_{1}$ and $h_{v_{1}}\left[S_{1}\right] \subseteq Q_{2}, Q_{2} \in \alpha$. Thus, by Lemma $2.10 Q_{2} \in p_{2}$, so by Lemma $2.9 p_{2}$ is minimal in $S_{2}$. Given any $u \in S\left(\begin{array}{l}2 \\ 1\end{array}\right), h_{u}\left[S_{2}\right] \subseteq S_{1}$, and so $h_{u}\left(p_{2}\right) \in \beta S_{1}$. It thus suffices to show that $h_{u}\left(p_{2}\right) \leq p_{1}$. If $u \in U_{2,1}$, then $h_{u}$ is the identity on $S_{1}$, so $h_{u}\left(p_{2}\right) \leq h_{u}\left(p_{1}\right)=p_{1}$. Now assume that $u \in U_{2,0}$ and pick $t \in A$ and $e \in E$ such that $u=t\left(e, \nu_{0}\right)$. For $w \in S_{1}, h_{u}(w)=h_{t}(w)$, and so $h_{u}\left(p_{1}\right)=h_{t}\left(p_{1}\right)=p_{0}$. Also, 
TABLE 1.

\begin{tabular}{|c|c|c|c|c|c|c|c|}
\hline$u$ & $x: p_{n-1}$ & $\delta_{1}$ & $\delta_{2}$ & $\delta_{3}$ & $\ldots$ & $\delta_{n-2}$ & $\delta_{n-1}$ \\
\hline$u_{n, n-1}$ & $p_{n-1}$ & & & & & & \\
\hline$u_{n, n-2}$ & $p_{n-2}$ & $\eta_{1}$ & & & & & \\
\hline$u_{n, n-3}$ & $p_{n-2}$ & $\gamma_{1}$ & $\eta_{2}$ & & & & \\
\hline$u_{n, n-4}$ & $p_{n-2}$ & $\gamma_{1}$ & $\gamma_{2}$ & $\eta_{3}$ & & & \\
\hline$\vdots$ & : & $\vdots$ & $\vdots$ & : & $\ddots$ & & \\
\hline$u_{n, 1}$ & $p_{n-2}$ & $\gamma_{1}$ & $\gamma_{2}$ & $\gamma_{3}$ & $\ldots$ & $\eta_{n-2}$ & \\
\hline$u_{n, 0}$ & $p_{n-2}$ & $\gamma_{1}$ & $\gamma_{2}$ & $\gamma_{3}$ & . & $\gamma_{n-2}$ & $\eta_{n-1}$ \\
\hline
\end{tabular}

by Lemma2.4(a) $h_{t v_{0}} \circ h_{v_{1}}$ is the identity on $W_{1}$. So $h_{u}(\alpha)=h_{t\left(e, \nu_{0}\right)}\left(h_{v_{1}}\left(p_{1}\right)\right)=p_{1}$. Therefore $h_{u}\left(p_{1} \alpha\right)=p_{0} p_{1}=p_{1}$ and $h_{u}\left(\alpha p_{1}\right)=p_{1} p_{0}=p_{1}$. Since $p_{2} \leq_{R} p_{1} \alpha$, $h_{u}\left(p_{2}\right) \leq_{R} h_{u}\left(p_{1} \alpha\right)=p_{1}$. Since $p_{2} \leq_{L} \alpha p_{1}, h_{u}\left(p_{2}\right) \leq_{L} h_{u}\left(\alpha p_{1}\right)=p_{1}$.

We now proceed to an inductive construction. Let $n \in \mathbb{N}$ with $n \geq 2$.

We shall introduce elements ( $\operatorname{such}$ as $\eta_{i}$ or $\gamma_{i}$ ) which depend on $n$ as well as on $i$. However, in an effort to reduce the number of subscripts used, we shall not indicate the dependence on $n$ in the notation.

We make the inductive assumption that we have chosen $p_{i}$ for $i \in\{0,1,2, \ldots n\}$, $\eta_{i}, \eta_{i}^{\prime}, \delta_{i}$, and $\delta_{i}^{\prime}$ for $i \in\{1,2,3, \ldots, n-1\}$, and $\gamma_{i}$ and $\gamma_{i}^{\prime}$ for $i \in\{2,3, \ldots, n-2\}$, if any, so that the following hypotheses are satisfied:

(a) For each $i \in\{0,1, \ldots, n\}, p_{i}$ is a minimal idempotent of $\beta S_{i}$.

(b) For each $i \in\{1,2, \ldots, n\}, p_{i} \leq p_{i-1}$ and $h_{u}\left(p_{i}\right)=p_{i-1}$ for every $u \in$ $S\left(\begin{array}{c}i \\ i-1\end{array}\right)$.

(c) For every $i \in\{1,2, \ldots, n-1\}, \eta_{i}$ and $\eta_{i}^{\prime}$ are minimal idempotents in $\beta W_{n-1}$.

(d) For every $i \in\{1,2, \ldots, n-1\}, \eta_{i} \leq_{L} p_{n-1}$ and $\eta_{i}^{\prime} \leq_{R} p_{n-1}$.

(e) For $i \in\{1,2, \ldots, n-1\}, \delta_{i}=h_{w_{n, n-i-1}}\left(\eta_{i}\right), \delta_{i}^{\prime}=h_{w_{n, n-i-1}}\left(\eta_{i}^{\prime}\right)$,

$$
\begin{aligned}
& p_{n} \leq_{R} p_{n-1} \delta_{1} \cdots \delta_{n-1}, \text { and } \\
& p_{n} \leq_{L} \delta_{n-1}^{\prime} \cdots \delta_{1}^{\prime} p_{n-1} .
\end{aligned}
$$

(f) For every $i \in\{1,2, \ldots, n-2\}$,

$$
\begin{aligned}
& \eta_{i} \leq_{R} \gamma_{i} \cdots \gamma_{n-2} \eta_{n-1} \text { and } \\
& \eta_{i}^{\prime} \leq_{L} \eta_{n-1}^{\prime} \gamma_{n-2}^{\prime} \cdots \gamma_{i}^{\prime} .
\end{aligned}
$$

(g) For every choice of $u_{n, i} \in U_{n, i}$ for $i \in\{0,1, \ldots, n-1\}$, the entry in the row labeled by $u$ and the column labeled by $x$ in Tables 1 and 2 is $h_{u}(x)$.

We observe that these assumptions do hold if $n=2$, with $\eta_{1}=\eta_{1}^{\prime}=p_{1}$. To verify hypothesis (c) we need to show that $p_{1}$ is minimal in $\beta W_{1}$. Since $S_{1}$ is a right ideal of $W_{1}$, we have that $\beta S_{1}$ is a right ideal of $\beta W_{1}$ and so contains a minimal right ideal of $\beta W_{1}$. Therefore by [10, Theorem 1.65] $K\left(\beta S_{1}\right)=\beta S_{1} \cap K\left(\beta W_{1}\right)$. For hypothesis (e), note that $\delta_{1}=\delta_{1}^{\prime}=\alpha$. Hypothesis (f) is vacuous, and we have already verified the table entries of hypothesis $(\mathrm{g})$.

Note that since $h_{w_{n, n-i-1}}\left[W_{n-1}\right] \subseteq W_{n}$, one has that each $\delta_{i} \in \beta W_{n}$. Also, since $h_{u}\left[W_{n}\right] \subseteq W_{n-1}$ for each $u \in S\left(\begin{array}{c}n \\ n-1\end{array}\right)$, we have that each $\gamma_{i} \in \beta W_{n-1}$. 
TABLE 2 .

\begin{tabular}{|c|c|c|c|c|c|c|}
\hline$u$ & $x: \delta_{n-1}^{\prime}$ & $\delta_{n-2}^{\prime}$ & $\delta_{3}^{\prime}$ & $\delta_{2}^{\prime}$ & $\delta_{1}^{\prime}$ & $p_{n-1}$ \\
\hline$u_{n, n-1}$ & & & & & & $p_{n-1}$ \\
\hline$u_{n, n-2}$ & & & & & $\eta_{1}^{\prime}$ & $p_{n-2}$ \\
\hline$u_{n, n-3}$ & & & & $\eta_{2}^{\prime}$ & $\gamma_{1}^{\prime}$ & $p_{n-2}$ \\
\hline$u_{n, n-4}$ & & & $\eta_{3}^{\prime}$ & $\gamma_{2}^{\prime}$ & $\gamma_{1}^{\prime}$ & $p_{n-2}$ \\
\hline$u_{n, 1}$ & & $\eta_{n-2}^{\prime}$ & $\gamma_{3}^{\prime}$ & $\gamma_{2}^{\prime}$ & $\gamma_{1}^{\prime}$ & $p_{n-2}$ \\
\hline$u_{n, 0}$ & $\eta_{n-1}^{\prime}$ & $\gamma_{n-2}^{\prime}$ & $\gamma_{3}^{\prime}$ & $\gamma_{2}^{\prime}$ & $\gamma_{1}^{\prime}$ & $p_{n-2}$ \\
\hline
\end{tabular}

By assumption (e), $p_{n} \leq_{R} p_{n-1} \delta_{1} \cdots \delta_{n-1}$. So there is some $x \in \beta W_{n}$ such that $p_{n-1} \delta_{1} \cdots \delta_{n-1} x=p_{n}=p_{n} p_{n} \in p_{n} \beta W$. So

$$
\left\{x \in \beta W_{n}: p_{n-1} \delta_{1} \cdots \delta_{n-1} x \leq_{R} p_{n}\right\}
$$

is nonempty and is therefore a right ideal of $\beta W_{n}$. By Lemma 2.7, we can choose a minimal idempotent $\mu_{n}$ of $\beta W_{n}$ which is in this right ideal and in the left ideal $\beta W_{n} p_{n}$ of $\beta W_{n}$.

Now let $i \in\{2,3, \ldots, n-1\}$. Note that $\delta_{i} \cdots \delta_{n-1} \mu_{n}=\delta_{i} \cdots \delta_{n-1} \mu_{n} \mu_{n}$, so

$$
\left\{x \in \beta W_{n}: p_{n-1} \delta_{1} \delta_{2} \cdots \delta_{i-1} x \leq_{R} p_{n} \text { and } x \leq_{R} \delta_{i} \cdots \delta_{n-1} \mu_{n}\right\}
$$

is nonempty, because it contains $\delta_{i} \cdots \delta_{n-1} \mu_{n}$. It is therefore a right ideal of $\beta W_{n}$, and we can choose a minimal idempotent $\mu_{i}$ of $\beta W_{n}$ which is in this right ideal and is also in the left ideal $\beta W_{n} p_{n}$ of $\beta W_{n}$.

Similarly, $\left\{x \in \beta W_{n}: p_{n-1} x \leq_{R} p_{n}\right.$ and $\left.x \leq_{R} \delta_{1} \cdots \delta_{n-1} \mu_{n}\right\}$ is nonempty because $\delta_{1} \cdots \delta_{n-1} \mu_{n}$ is a member, and thus we may choose a minimal idempotent $\mu_{1}$ of $\beta W_{n}$ which is in this right ideal of $\beta W_{n}$ and also in the left ideal $\beta W_{n} p_{n}$.

Thus we have chosen minimal idempotents $\mu_{1}, \mu_{2}, \ldots, \mu_{n}$ in $\beta W_{n}$ which satisfy the following conditions:

$$
\begin{aligned}
\mu_{i} & \leq_{L} p_{n} \text { for all } i \in\{1,2, \ldots, n\} \\
p_{n-1} \delta_{1} \cdots \delta_{i-1} \mu_{i} & \leq_{R} p_{n} \text { for all } i \in\{2,3, \ldots, n\} \\
p_{n-1} \mu_{1} & \leq_{R} p_{n} ; \text { and } \\
\mu_{i} & \leq_{R} \delta_{i} \cdots \delta_{n-1} \mu_{n} \text { for all } i \in\{1,2,3, \ldots, n-1\} .
\end{aligned}
$$

By a left-right switch of these arguments, we can choose minimal idempotents $\mu_{1}^{\prime}, \mu_{2}^{\prime}, \ldots, \mu_{n}^{\prime}$ in $\beta W_{n}$ which satisfy the following conditions:

$$
\begin{aligned}
\mu_{i}^{\prime} & \leq_{R} p_{n} \text { for all } i \in\{1,2, \ldots, n\} \\
\mu_{i}^{\prime} \delta_{i-1}^{\prime} \cdots \delta_{1}^{\prime} p_{n-1} & \leq_{L} p_{n} \text { for all } i \in\{2,3, \ldots, n\} \\
\mu_{1}^{\prime} p_{n-1} & \leq_{L} p_{n} ; \text { and } \\
\mu_{i}^{\prime} & \leq_{L} \mu_{n}^{\prime} \delta_{n-1}^{\prime} \cdots \delta_{i}^{\prime} \text { for all } i \in\{1,2,3, \ldots, n-1\} .
\end{aligned}
$$

(While $\beta W$ is right topological and not left topological, all of the algebraic facts that we are using in this proof are valid from both sides.)

For $i \in\{1,2, \ldots, n\}$, let $\epsilon_{i}=h_{w_{n+1, n-i}}\left(\mu_{i}\right)$, let $\epsilon_{i}^{\prime}=h_{w_{n+1, n-i}}\left(\mu_{i}^{\prime}\right)$, and note that $\epsilon_{i}, \epsilon_{i}^{\prime} \in W_{n+1}$. Then $p_{n} \epsilon_{1} \cdots \epsilon_{n} \beta W_{n+1}$ and $\beta W_{n+1} \epsilon_{n}^{\prime} \cdots \epsilon_{1}^{\prime} p_{n}$ are respectively right and left ideals of $\beta W_{n+1}$. By Lemma 2.7 pick a minimal idempotent $p_{n+1}$ of $\beta W_{n+1}$ such that

$$
p_{n+1} \in p_{n} \epsilon_{1} \cdots \epsilon_{n} \beta W_{n+1} \cap \beta W_{n+1} \epsilon_{n}^{\prime} \cdots \epsilon_{1}^{\prime} p_{n} .
$$


TABLE 3 .

\begin{tabular}{c|r|c|c|c|c|c|c|}
$u$ & $x: p_{n}$ & $\epsilon_{1}$ & $\epsilon_{2}$ & $\epsilon_{3}$ & $\cdots$ & $\epsilon_{n-1}$ & $\epsilon_{n}$ \\
\hline$u_{n+1, n}$ & $p_{n}$ & & & & & & \\
$u_{n+1, n-1}$ & $p_{n-1}$ & $\mu_{1}$ & & & & & \\
$u_{n+1, n-2}$ & $p_{n-1}$ & $\delta_{1}$ & $\mu_{2}$ & & & & \\
$u_{n+1, n-3}$ & $p_{n-1}$ & $\delta_{1}$ & $\delta_{2}$ & $\mu_{3}$ & & & \\
$\vdots$ & $\vdots$ & $\vdots$ & $\vdots$ & $\vdots$ & $\ddots$ & & \\
$u_{n+1,1}$ & $p_{n-1}$ & $\delta_{1}$ & $\delta_{2}$ & $\delta_{3}$ & $\cdots$ & $\mu_{n-1}$ & \\
$u_{n+1,0}$ & $p_{n-1}$ & $\delta_{1}$ & $\delta_{2}$ & $\delta_{3}$ & $\cdots$ & $\delta_{n-1}$ & $\mu_{n}$
\end{tabular}

TABLE 4 .

\begin{tabular}{|c|c|c|c|c|c|c|c|}
\hline$u$ & $x: \epsilon_{n}^{\prime}$ & $\epsilon_{n-1}^{\prime}$ & $\ldots$ & $\epsilon_{3}^{\prime}$ & $\epsilon_{2}^{\prime}$ & $\epsilon_{1}^{\prime}$ & $p_{n}$ \\
\hline$u_{n+1, n}$ & & & & & & & $p_{n}$ \\
\hline$u_{n+1, n-1}$ & & & & & & $\mu_{1}^{\prime}$ & $p_{n-1}$ \\
\hline$u_{n+1, n-2}$ & & & & & $\mu_{2}^{\prime}$ & $\delta_{1}^{\prime}$ & $p_{n-1}$ \\
\hline$u_{n+1, n-3}$ & & & & $\mu_{3}^{\prime}$ & $\delta_{2}^{\prime}$ & $\delta_{1}^{\prime}$ & $p_{n-1}$ \\
\hline$:$ & & &. & & & $\cdot$ & $:$ \\
\hline$u_{n+1,1}$ & & $\mu_{n-1}^{\prime}$ & & $\delta_{3}^{\prime}$ & $\delta_{2}^{\prime}$ & $\delta_{1}^{\prime}$ & $p_{n-1}$ \\
\hline$u_{n+1,0}$ & $\mu_{n}^{\prime}$ & $\delta_{n-1}^{\prime}$ & & $\delta_{3}^{\prime}$ & $\delta_{2}^{\prime}$ & $\delta_{1}^{\prime}$ & $p_{n-1}$ \\
\hline
\end{tabular}

We claim that $p_{n+1}$ is a minimal idempotent of $\beta S_{n+1}$. By $(*), p_{n-1} \mu_{1} \leq_{R} p_{n}$, so by Lemma 2.11, $Q_{n} \in \mu_{1}$. Since $h_{w_{n+1, n-1}}\left[Q_{n}\right] \subseteq Q_{n+1}, Q_{n+1} \in \epsilon_{1}$, and so, by Lemma 2.10, $Q_{n+1} \in p_{n+1}$. Consequently, by Lemma $2.9 p_{n+1}$ is minimal in $\beta S_{n+1}$.

We now claim that the induction hypotheses are satisfied for $n+1$ with $\eta_{i}, \eta_{i}^{\prime}$, $\delta_{i}, \delta_{i}^{\prime}, \gamma_{i}$, and $\gamma_{i}^{\prime}$ replaced by $\mu_{i}, \mu_{i}^{\prime}, \epsilon_{i}, \epsilon_{i}^{\prime}, \delta_{i}$, and $\delta_{i}^{\prime}$, respectively. That is, we claim that

(a) For each $i \in\{0,1, \ldots, n+1\}, p_{i}$ is a minimal idempotent of $\beta S_{i}$.

(b) For each $i \in\{1,2, \ldots, n+1\}, p_{i} \leq p_{i-1}$ and $h_{u}\left(p_{i}\right)=p_{i-1}$ for every $u \in S\left(\begin{array}{c}i \\ i-1\end{array}\right)$.

(c) For every $i \in\{1,2, \ldots, n\}, \mu_{i}$ and $\mu_{i}^{\prime}$ are minimal idempotents in $\beta W_{n}$.

(d) For every $i \in\{1,2, \ldots, n\}, \mu_{i} \leq_{L} p_{n}$ and $\mu_{i}^{\prime} \leq_{R} p_{n}$.

(e) For $i \in\{1,2, \ldots, n\}, \epsilon_{i}=h_{w_{n+1, n-i}}\left(\mu_{i}\right), \epsilon_{i}^{\prime}=h_{w_{n+1, n-i}}\left(\mu_{i}^{\prime}\right)$,

$$
\begin{aligned}
& p_{n+1} \quad \leq_{R} p_{n} \epsilon_{1} \cdots \epsilon_{n} \text { and } \\
& p_{n+1} \leq_{L} \epsilon_{n}^{\prime} \cdots \epsilon_{1}^{\prime} p_{n} .
\end{aligned}
$$

(f) For every $i \in\{1,2, \ldots, n-1\}$,

$$
\begin{array}{ll}
\mu_{i} & \leq_{R} \delta_{i} \cdots \delta_{n-1} \mu_{n} \text { and } \\
\mu_{i}^{\prime} & \leq_{L} \mu_{n}^{\prime} \delta_{n-1}^{\prime} \cdots \delta_{i}^{\prime} .
\end{array}
$$

(g) For every choice of $u_{n+1, i} \in U_{n+1, i}$ for $i \in\{0,1, \ldots, n\}$, the entry in the row labeled by $u$ and the column labeled by $x$ in Tables 3 and 4 is $h_{u}(x)$. 
All of these conclusions can be easily verified except for $(\mathrm{g})$ and the assertion in (b) that $h_{u}\left(p_{n+1}\right)=h_{u}\left(p_{n}\right)$ for all $u \in S\left(\begin{array}{c}n \\ n-1\end{array}\right)$. We show first that this latter assertion follows from statement $(\mathrm{g})$.

For any $i \in\{0,1, \ldots, n\}, h_{u_{n+1}, i}\left(p_{n+1}\right) \in \beta S_{n}$ and $p_{n}$ is minimal in $\beta S_{n}$, so it suffices to show that $h_{u_{n+1, i}}\left(p_{n+1}\right) \leq p_{n}$. Since $p_{n+1} \leq p_{n}$ and $h_{u_{n+1, n}}$ is the identity on $W_{n}$, we have that $h_{u_{n+1, n}}\left(p_{n+1}\right) \leq h_{u_{n+1, n}}\left(p_{n}\right)=p_{n}$.

Now let $i \in\{0,1, \ldots, n-1\}$ and let $u=u_{n+1, i}$. We have $p_{n+1} \leq_{R} p_{n} \epsilon_{1} \cdots \epsilon_{n-i}$ and so $h_{u}\left(p_{n+1}\right) \leq_{R} h_{u}\left(p_{n} \epsilon_{1} \cdots \epsilon_{n-i}\right)$, and by $(*)$ and Table $3, h_{u}\left(p_{n} \epsilon_{1} \cdots \epsilon_{n-i}\right) \leq_{R}$ $p_{n}$. Also $p_{n+1} \leq_{L} \epsilon_{n-i}^{\prime} \cdots \epsilon_{1}^{\prime} p_{n}$ so $h_{u}\left(p_{n+1}\right) \leq_{L} h_{u}\left(\epsilon_{n-i}^{\prime} \cdots \epsilon_{1}^{\prime} p_{n}\right)$, and by $(* *)$ and Table $4, h_{u}\left(\epsilon_{n-i}^{\prime} \cdots \epsilon_{1}^{\prime} p_{n}\right) \leq_{L} p_{n}$.

It thus suffices to verify the entries of Table 3 and Table 4 . We shall write out the verification for Table 3 . The verification for Table 4 follows by a left-right switch of the arguments. To this end, let a choice of $u_{n+1, i} \in U_{n+1, i}$ for $i \in\{0,1, \ldots, n\}$ be given.

We have that $h_{u_{n+1, n}}$ is the identity on $S_{n}$, so $h_{u_{n+1, n}}\left(p_{n}\right)=p_{n}$. For $i \in\{0,1$, $\ldots, n-1\}, h_{u_{n+1, i}}=h_{u_{n, i}}$ on $S_{n}$ so $h_{u_{n+1, i}}\left(p_{n}\right)=h_{u_{n, i}}\left(p_{n}\right)=p_{n-1}$ by hypothesis (b).

The diagonal entries are correct because $\epsilon_{i}=h_{w_{n+1, n-i}}\left(\mu_{i}\right)$ for $i \in\{1,2, \ldots, n\}$ and $h_{u_{n+1, n-i}} \circ h_{w_{n+1, n-i}}$ is the identity on $W_{n}$ by Lemma 2.4(a).

Let $k \in\{1,2, \ldots, n-1\}$, let $i \in\{0,1, \ldots, n-k-1\}$, and let $u \in U_{n+1, i}$. To finish the proof we need to show that $h_{u}\left(\epsilon_{k}\right)=\delta_{k}$. Now $\epsilon_{k}=h_{w_{n+1, n-k}}\left(\mu_{k}\right)$, so we are showing that $h_{u}\left(h_{w_{n+1, n-k}}\left(\mu_{k}\right)\right)=\delta_{k}$. Since $i<n-k$, we have by Lemma 2.4(b) that $h_{u}\left(h_{w_{n+1, n-k}}\left(\mu_{k}\right)\right)=h_{w_{n, n-k-1}}\left(h_{u}\left(\mu_{k}\right)\right)$. So it suffices to show that $h_{w_{n, n-k-1}}\left(h_{u}\left(\mu_{k}\right)\right)=\delta_{k}$. Now $h_{w_{n, n-k-1}}\left(\eta_{k}\right)=\delta_{k}$ by hypothesis (e), so it suffices to show that $h_{u}\left(\mu_{k}\right)=\eta_{k}$. Also, since $h_{u}\left(\mu_{k}\right)$ and $\eta_{k}$ are idempotents in $\beta W_{n-1}$ and $\eta_{k}$ is minimal in $\beta W_{n-1}$, it suffices to show that $h_{u}\left(\mu_{k}\right) \leq \eta_{k}$.

Now $\mu_{k} \leq_{L} p_{n}$ by $(*)$, so $h_{u}\left(\mu_{k}\right) \leq_{L} h_{u}\left(p_{n}\right)=p_{n-1}$, the equality holding by hypothesis (b). Since $\eta_{k} \leq_{L} p_{n-1}$ by hypothesis (d), we have by Lemma 2.6(d) that $h_{u}\left(\mu_{k}\right) \leq_{L} \eta_{k}$.

It remains to show that $h_{u}\left(\mu_{k}\right) \leq_{R} \eta_{k}$. We have by $(*)$ that $\mu_{k} \leq_{R} \delta_{k} \cdots \delta_{n-1} \mu_{n}$. If $i=n-k-1$, we have that $h_{u}\left(\mu_{k}\right) \leq_{R} h_{u}\left(\delta_{k}\right)=\eta_{k}$ by hypothesis (g), so assume that $i<n-k-1$. Then $h_{u}\left(\mu_{k}\right) \leq_{R} h_{u}\left(\delta_{k}\right) \cdots h_{u}\left(\delta_{n-i-1}\right)=\gamma_{k} \cdots \gamma_{n-i-2} \eta_{n-i-1}$, the equality holding by hypothesis (g). If $i=0$, we have directly that $h_{u}\left(\mu_{k}\right) \leq_{R}$ $\gamma_{k} \cdots \gamma_{n-2} \eta_{n-1}$. Otherwise $\eta_{n-i-1} \leq_{R} \gamma_{n-i-1} \cdots \gamma_{n-2} \eta_{n-1}$ by hypothesis (f), so again $h_{u}\left(\mu_{k}\right) \leq_{R} \gamma_{k} \cdots \gamma_{n-2} \eta_{n-1}$. Also $\eta_{k} \leq_{R} \gamma_{k} \cdots \gamma_{n-2} \eta_{n-1}$ by hypothesis (f). Now $\eta_{n-1} \in K\left(\beta W_{n-1}\right)$ and $\gamma_{k} \cdots \gamma_{n-2} \in \beta W_{n-1}$, so $\gamma_{k} \cdots \gamma_{n-2} \eta_{n-1} \in$ $K\left(\beta W_{n-1}\right)$, and thus by Lemma 2.6 (c), $h_{u}\left(\mu_{k}\right) \leq_{R} \eta_{k}$.

One might expect to be able to omit $p_{0}$ and start in Theorem 2.12 with $p_{1}$ as any minimal idempotent in $\beta S_{1}$. It is a consequence of Theorem 3.6 below that one cannot.

The following lemmas will be useful in the next section.

Lemma 2.13. Let $k, m \in \omega$ with $k<m$ and let $u \in S\left(\begin{array}{c}m \\ k\end{array}\right)$. There exist $r \in$ $S\left(\begin{array}{c}m \\ m-1\end{array}\right)$ and $s \in S\left(\begin{array}{c}m-1 \\ k\end{array}\right)$ such that for all $w \in W_{m}, h_{s}\left(h_{r}(w)\right)=h_{u}(w)$. 
Proof. Either $u(j) \in A$ for some $j \in\{0,1, \ldots, m-1\}$ or else there exists $t \in\{0,1$, $\ldots, k-1\}$ such that elements of $D \times\left\{\nu_{t}\right\}$ occur more than once in $u$. In the second case, let $t$ be the smallest index for which this happens. Then $u(t) \in E \times\left\{\nu_{t}\right\}$ and there exists $j>t$ such that $u(j) \in D \times\left\{\nu_{t}\right\}$. In either case, we define $r$ and $s$ as follows for $i \in\{0,1, \ldots, m-1\}$ and $l \in\{0,1, \ldots, m-2\}$ :

$$
r(i)=\left\{\begin{array}{cl}
v_{i} & \text { if } i<j, \\
u(j) & \text { if } i=j, \\
v_{i-1} & \text { if } j<i,
\end{array} \text { and } s(l)=\left\{\begin{array}{cl}
u(l) & \text { if } l<j, \\
u(l+1) & \text { if } j \leq l .
\end{array}\right.\right.
$$

Lemma 2.14. Let $n \in \mathbb{N}$ and let $p_{0} \in \beta W_{0}$, and for each $i \in\{1,2, \ldots, n\}$, let $p_{i} \in \beta S_{i}$. Assume that for each $i \in\{0,1, \ldots, n-1\}$ and each $u \in S\left(\begin{array}{c}i+1 \\ i\end{array}\right)$, $h_{u}\left(p_{i+1}\right)=p_{i}$. Then for each $k<m$ in $\{0,1, \ldots, n\}$ and each $u \in S\left(\begin{array}{c}m \\ k\end{array}\right), h_{u}\left(p_{m}\right)=$ $p_{k}$.

Proof. We proceed by induction on $n$, the case $n=1$ being trivial. So let $n \in \mathbb{N}$ and assume the lemma is true for $n$. Let $p_{0}, p_{1}, \ldots, p_{n+1}$ be as in the statement of the lemma, let $k<m$ in $\{0,1, \ldots, n+1\}$, and let $u \in S\left(\begin{array}{c}m \\ k\end{array}\right)$. By Lemma 2.13 pick $r \in S\left(\begin{array}{c}m \\ m-1\end{array}\right)$ and $s \in S\left(\begin{array}{c}m-1 \\ k\end{array}\right)$ such that for all $w \in S_{m}, h_{s}\left(h_{r}(w)\right)=h_{u}(w)$. Since $p_{m} \in \beta S_{m}, h_{u}\left(p_{m}\right)=h_{s}\left(h_{r}\left(p_{m}\right)\right)$. Then $h_{r}\left(p_{m}\right)=p_{m-1}$ and by the induction hypothesis, $h_{s}\left(p_{m-1}\right)=p_{k}$.

\section{Extending the Graham-Rothschild Parameter Sets Theorem}

Theorem 3.2 is the main Ramsey Theoretic result of this paper. In order to state it precisely, we need to formalize the notion of "tree". Recall that an ordinal is the set of its predecessors, so that, if $n \in \mathbb{N}$, then $n=\{0,1, \ldots, n-1\}$.

Definition 3.1. Let $X$ be a set.

(1) $T$ is a tree in $X$ if and only if

(a) $T$ is a nonempty set of functions,

(b) for each $f \in T$, $\operatorname{dom}(f) \in \omega$ and $\operatorname{ran}(f) \subseteq X$, and

(c) for each $f \in T$, if $\operatorname{dom}(f)=n>0$, then $f_{\mid n-1} \in T$.

(2) If $T$ is a tree in $X$ and $n \in \omega$, then $T_{n}=\{f \in T: \operatorname{dom}(f)=n\}$.

(3) If $T$ is a tree in $X$ and $f \in T$, then $B_{f}=\{x \in X: f \cup\{(n, x)\} \in T\}$.

(4) The sequence $\left\langle w_{n}\right\rangle_{n=0}^{\infty}$ is a path through $T$ if and only if for each $n \in \omega$, $\left\{\left(k, w_{k}\right): k \in\{0,1, \ldots, n\}\right\} \in T$.

The empty function is a "root" for the tree, and $B_{f}$ is the set of successors to the "node" $f$. Consider, for example, the following diagram of a tree $T$ :

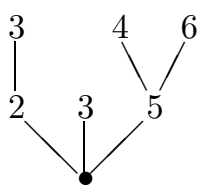


Then formally

$$
\begin{aligned}
T & =\{\emptyset,\{(0,2)\},\{(0,3)\},\{(0,5)\},\{(0,2),(1,3)\},\{(0,5),(1,4)\},\{(0,5),(1,6)\}\}, \\
B_{\emptyset} & =\{2,3,5\} \text { and } B_{\{(0,5)\}}=\{4,6\} .
\end{aligned}
$$

Note that if $w \in S_{m}$ and $u \in S\left(\begin{array}{c}m \\ m\end{array}\right)$, then $w\langle u\rangle=w$.

Condition (2) of the following theorem says that each path through $T$ satisfies the conclusion of Corollary 3.3 below and that the monochrome colors are invariant from path to path.

Theorem 3.2. Assume that, for each $n \in \omega, S_{n}$ has been finitely colored and that, for each $n \in \omega$ and each $i \in\{0,1, \ldots, n\}, H_{n, i}$ is a finite subset of $S\left(\begin{array}{c}n \\ i\end{array}\right)$. Then there is a tree $T$ in $W$ such that

(1) for each $n \in \omega$ and each $f \in T_{n}, B_{f}$ is a monochrome central subset of $S_{n}$ and

(2) for any $m \in \omega$, the intersection of $S_{m}$ with

$$
\left\{\prod_{n \in F} f(n)\left\langle u_{n}\right\rangle: f \in T, \emptyset \neq F \subseteq \operatorname{dom}(f) \text {, and for all } n \in F, u_{n} \in \bigcup_{i=0}^{\min F} H_{n, i}\right\}
$$

is monochrome.

Proof. Pick a sequence of idempotents $\left\langle p_{n}\right\rangle_{n=0}^{\infty}$ as guaranteed by Theorem 2.12, For $n \in \omega$, choose a monochrome set $C_{n} \subseteq S_{n}$ such that $C_{n} \in p_{n}$.

Let $T_{0}=\{\emptyset\}$ and let $V_{\emptyset, 0}=C_{0}$. Inductively, let $k \in \omega$ and assume that for $l \in\{0,1, \ldots, k\}$ we have defined $T_{l}$ and for each $f \in T_{l}$ and each $i \in\{0,1, \ldots, l\}$ we have defined $V_{f, i}$ such that

(1) $\bigcup_{l=0}^{k} T_{l}$ is a tree in $W$;

(2) if $l \in\{0,1, \ldots, k-1\}$ and $f \in T_{l}$, then $B_{f} \subseteq C_{l}$ and $B_{f} \in p_{l}$;

(3) if $l \in\{0,1, \ldots, k\}, f \in T_{l}$, and $j \in\{0,1, \ldots, l\}$, then $V_{f, j} \subseteq C_{j}$ and $V_{f, j} \in p_{j}$

(4) if $l \in\{0,1, \ldots, k-1\}, i \in\{0,1, \ldots, l\}, f \in T_{l}$, and $u \in H_{l, i}$, then $B_{f} \subseteq$ $h_{u}{ }^{-1}\left[V_{f, i}\right]$

(5) if $l \in\{1,2, \ldots, k\}, m \in\{0,1, \ldots, l-1\}, j \in\{0,1, \ldots, m\}, g \in T_{l}$, and $f=g_{\mid m}$, then $V_{g, j} \subseteq V_{f, j}$;

(6) if $l \in\{1,2, \ldots, k\}, i \in\{0,1, \ldots, l\}, j \in\{0,1, \ldots, i\}, g \in T_{l}, f=g_{\mid l-1}$, and $u \in H_{l-1, i}$, then $h_{u}(g(l-1)) V_{g, j} \subseteq V_{f, i}$; and

(7) if $l \in\{1,2, \ldots, k\}, j \in\{0,1, \ldots, l\}, i \in\{0,1, \ldots, j\}, g \in T_{l}, f=g_{\mid l-1}$, and $u \in H_{l-1, i}$, then $h_{u}(g(l-1)) V_{g, j} \subseteq V_{f, j}$.

These hypotheses are valid for $k=0$, all except (1) and (3) vacuously.

Now for $f \in T_{k}$ and $i \in\{0,1, \ldots, k\}$, let

$$
U_{f, i}=V_{f, i} \cap \bigcap_{j=i}^{k}\left\{w \in W: w^{-1} V_{f, j} \in p_{j}\right\} \cap \bigcap_{j=0}^{i}\left\{w \in W: w^{-1} V_{f, i} \in p_{j}\right\} .
$$

(We include the $j=i$ term in both intersections to avoid worrying about $i=0$ or $i=k$.) Given $j \in\{i, i+1, \ldots, k\}$ we have that $p_{j}=p_{i} p_{j}$ and $V_{f, j} \in p_{j}$, so $\left\{w \in W: w^{-1} V_{f, j} \in p_{j}\right\} \in p_{i}$. Given $j \in\{0,1, \ldots, i\}$, we have that $p_{i}=p_{i} p_{j}$ and $V_{f, i} \in p_{i}$, so $\left\{w \in W: w^{-1} V_{f, i} \in p_{j}\right\} \in p_{i}$. Consequently $U_{f, i} \in p_{i}$. 
Given $i \in\{0,1, \ldots, k\}, f \in T_{k}$, and $u \in S\left(\begin{array}{c}k \\ i\end{array}\right)$, we have by Lemma 2.14 that $h_{u}\left(p_{k}\right)=p_{i}$, and so $h_{u}{ }^{-1}\left[U_{f, i}\right] \in p_{k}$. For $f \in T_{k}$, let

$$
D_{f}=C_{k} \cap \bigcap\left\{h_{u}{ }^{-1}\left[U_{f, i}\right]: i \in\{0,1, \ldots, k\} \text { and } u \in H_{k, i}\right\},
$$

and note that $D_{f} \in p_{k}$ because $H_{k, i}$ is finite. (In this and all similar expressions we take $C \cap \bigcap \emptyset=C$. Thus, if $\bigcup_{i=0}^{k} H_{k, i}=\emptyset$, then $D_{f}=C_{k}$.)

Now let $T_{k+1}=\left\{f \cup\left\{(k, x): f \in T_{k}\right.\right.$ and $\left.x \in D_{f}\right\}$. For $g \in T_{k+1}$, let $f=g_{\mid k}$, let $V_{g, k+1}=C_{k+1}$, and for $j \in\{0,1, \ldots, k\}$, let

$$
\begin{aligned}
V_{g, j}=V_{f, j} & \cap \bigcap\left\{\left(h_{u}(g(k))\right)^{-1} V_{f, i}: i \in\{j, j+1, \ldots, k\} \text { and } u \in H_{k, i}\right\} \\
& \cap \bigcap\left\{\left(h_{u}(g(k))\right)^{-1} V_{f, j}: i \in\{0,1, \ldots, j\} \text { and } u \in H_{k, i}\right\} .
\end{aligned}
$$

Then hypothesis (1) is satisfied directly, and given $f \in T_{l}$ we have that $B_{f}=D_{f}$, so hypothesis (2) holds.

To verify hypothesis (3), let $g \in T_{k+1}$ and let $j \in\{0,1, \ldots, k+1\}$. If $j=k+1$ we have that $V_{g, j}=C_{j} \in p_{j}$, so assume that $j \leq k$ and let $f=g_{\mid k}$. Then $f \in T_{k}$ and $V_{g, j} \subseteq V_{f, j} \subseteq C_{j}$ and $V_{f, j} \in p_{j}$. Note that $g(k) \in B_{f}=D_{f}$, so for $i \in\{0,1, \ldots, k\}$ and $u \in H_{k, i}, h_{u}(g(k)) \in U_{f, i}$. Thus if $i \leq j,\left(h_{u}(g(k))\right)^{-1} V_{f, j} \in p_{j}$ and if $i \geq j$, $\left(h_{u}(g(k))\right)^{-1} V_{f, i} \in p_{j}$, so $V_{g, j} \in p_{j}$.

Hypothesis (4) follows directly from the definition of $D_{f}$ for $f \in T_{k}$. Hypothesis (5) follows from the definition of $V_{g, j}$ for $g \in T_{k+1}$ and $j \in\{0,1, \ldots, k\}$ and the fact that hypothesis (5) holds at earlier stages. Hypotheses (6) and (7) follow directly from the definition of $V_{g, j}$ for $g \in T_{k+1}$ and $j \in\{0,1, \ldots, k\}$.

The induction being complete, we have from hypothesis (2) that the first conclusion of the theorem holds. To verify the second conclusion, we show that $\prod_{n \in F} f(n)\left\langle u_{n}\right\rangle \in C_{m}$ whenever $f \in T, \emptyset \neq F \subseteq \operatorname{dom}(f), \prod_{n \in F} f(n)\left\langle u_{n}\right\rangle \in S_{m}$, and for all $n \in F, u_{n} \in \bigcup_{i=0}^{\min F} H_{n, i}$. To do this, let $f \in T$. We show by induction on $|F|$ that if $\emptyset \neq F \subseteq \operatorname{dom}(f), k=\min F, g=f_{\mid\{0,1, \ldots, k-1\}}$, for each $n \in F$, $u_{n} \in \bigcup_{i=0}^{k} H_{n, i}$, and $\prod_{n \in F} f(n)\left\langle u_{n}\right\rangle \in S_{m}$, then $\prod_{n \in F} f(n)\left\langle u_{n}\right\rangle \in V_{g, m}$. (Since $V_{g, m} \subseteq C_{m}$ by hypothesis (3), this will suffice.)

Assume first that $F=\{k\}$. Then $h_{u_{k}}(f(k))=f(k)\left\langle u_{k}\right\rangle \in S_{m}$, so $u_{k} \in H_{k, m}$. Also, $w_{k} \in B_{g} \subseteq h_{u_{k}}{ }^{-1}\left[V_{g, m}\right]$ by hypothesis (4), so $h_{u_{k}}\left(w_{k}\right) \in V_{g, m}$ as required.

Assume that $|F|>1$. Let $r=\min (F \backslash\{k\})$, let $\hat{g}=f_{\{\{0,1, \ldots, r-1\}}$, and let $g^{*}=$ $f_{\mid\{0,1, \ldots, k\}}$. Pick $j$ such that $\prod_{n \in F \backslash\{k\}} f(n)\left\langle u_{n}\right\rangle \in S_{j}$. Note that $j \leq m \leq k<r$. Then by the induction hypothesis $\prod_{n \in F \backslash\{k\}} f(n)\left\langle u_{n}\right\rangle \in V_{\hat{g}, j}$ and by hypothesis (5), $V_{\hat{g}, j} \subseteq V_{g^{*}, j}$. Pick $i \in\{0,1, \ldots, k\}$ such that $u_{k} \in S\left(\begin{array}{c}k \\ i\end{array}\right)$. If $j \leq i$, then $h_{u_{k}}(f(k)) V_{g^{*}, j} \subseteq V_{g, i}$ by hypothesis (6), and so $\prod_{n \in F} f(n)\left\langle u_{n}\right\rangle \in V_{g, i}=V_{g, m}$. If $j \geq i$, then $h_{u_{k}}(f(k)) V_{g^{*}, j} \subseteq V_{g, j}$ by hypothesis (7), and so $\prod_{n \in F} f(n)\left\langle u_{n}\right\rangle \in$ $V_{g, j}=V_{g, m}$.

The fact that the set of successors to each node of the tree constructed in Theorem 3.2 is central means that that set of successors itself has very rich combinatorial structure as given by the Noncommutative Central Sets Theorem [10, Theorem 14.15]. Some of the more simply stated consequences of the centrality of $B_{f}$ hold 
because $\ell: S_{m} \rightarrow \mathbb{N}$ is a homomorphism and thus so is its continuous extension $\ell: \beta S_{m} \rightarrow \beta \mathbb{N}$. Therefore, by [10, Exercise 1.7.3 and Lemma 3.30], if $f \in T$, then $\ell\left[B_{f}\right]$ is central in $\mathbb{N}$. Thus, for example, there will be members of $B_{f}$ whose lengths form arbitrarily long arithmetic progressions.

Corollary 3.3. Assume that, for each $n \in \omega, S_{n}$ has been finitely colored and that, for each $n \in \omega$ and each $i \in\{0,1, \ldots, n\}, H_{n, i}$ is a finite subset of $S\left(\begin{array}{c}n \\ i\end{array}\right)$. Then, there exists a sequence $\left\langle w_{n}\right\rangle_{n<\omega}$ with each $w_{n} \in S_{n}$ such that for every $m \in \omega$,

$$
S_{m} \cap\left\{\prod_{n \in F} w_{n}\left\langle u_{n}\right\rangle: F \in \mathcal{P}_{f}(\omega) \text { and for all } n \in F, u_{n} \in \bigcup_{i=0}^{\min F} H_{n, i}\right\}
$$

is monochrome. (That is, the color of $\prod_{n \in F} w_{n}\left\langle u_{n}\right\rangle$ is determined solely by the number of parameters in $\prod_{n \in F} w_{n}\left\langle u_{n}\right\rangle$.)

Proof. Let $T$ be a tree as guaranteed by Theorem 3.2 and let $\left\langle w_{n}\right\rangle_{n=0}^{\infty}$ be any path through $T$.

Note that in the statement of Corollary 3.3 , the requirement $u_{n} \in \bigcup_{i=0}^{\min F} H_{i, n}$ cannot be replaced by the requirement that $u_{n} \in \bigcup_{i=0}^{n} H_{i, n}$. To see this, for each $n \in \omega$, let $r_{n}=n+1$ and define $\varphi_{n}: S_{n} \rightarrow\left\{1,2, \ldots, r_{n}\right\}$ by $\varphi_{n}(w) \equiv \ell(w) \bmod n$. Then given $w_{1}$ and $n$ greater than $\ell\left(w_{1}\right)$, one cannot have $\varphi_{n}\left(w_{1} w_{n}\right)=\varphi_{n}\left(w_{n}\right)$. (Recall that $w_{1}=w_{1}\left\langle v_{1}\right\rangle$ and $w_{n}=w_{n}\left\langle v_{1} \cdots v_{n}\right\rangle$.)

If one is only interested in the following corollary, one may prove it in a fashion similar to the proof of Theorem 3.2 .

Corollary 3.4. Let $m \in \mathbb{N}$. Suppose that we have a finite coloring of $\bigcup_{i=0}^{m} S_{i}$ and a finite subset $H$ of $\bigcup_{i=0}^{m} S\left(\begin{array}{c}m \\ i\end{array}\right)$. Then there exists a sequence $\left\langle w_{n}\right\rangle_{n=0}^{\infty}$ in $S_{m}$ such that for every $l \in\{0,1, \ldots, m\}$,

$$
S_{l} \cap\left\{\prod_{n \in F} w_{n}\left\langle u_{n}\right\rangle: F \in \mathcal{P}_{f}(\omega) \text { and for all } n \in F, u_{n} \in H\right\}
$$

is monochrome.

Proof. For each $n \in \omega$, we define a finite coloring of $S_{n}$ by stating that it coincides with the given coloring if $n \leq m$ and that it is the constant coloring if $n>m$. Choose any $a \in A$ and, for $n \in \mathbb{N}$, put $a^{n}=a \cdots a \in S\left(\begin{array}{l}n \\ 0\end{array}\right)$ and let $a^{0}=\emptyset$.

For each $n \in \omega$ and $i \in\{0,1, \ldots, m\}$, let $H_{m+n, i}=\left\{u a^{n}: u \in H \cap S\left(\begin{array}{c}m \\ i\end{array}\right)\right\}$. For all other values of $r$ and $i$, put $H_{r, i}=\emptyset$. Pick a sequence $\left\langle w_{n}^{\prime}\right\rangle_{n=0}^{\infty}$ as guaranteed by Corollary 3.3. For $n \in \omega$, let $s_{n}=v_{0} \cdots v_{m-1} a^{n}$ and let $w_{n}=h_{s_{n}}\left(w_{m+n}^{\prime}\right)$. To see that the sequence $\left\langle w_{n}\right\rangle_{n=0}^{\infty}$ is as required, let $l \in\{0,1, \ldots, m\}$. We shall show that

$$
S_{l} \cap\left\{\prod_{n \in F} w_{n}\left\langle u_{n}\right\rangle: F \in \mathcal{P}_{f}(\omega) \text { and for all } n \in F, u_{n} \in H\right\}
$$

is a subset of

$$
S_{l} \cap\left\{\prod_{k \in F^{\prime}} w_{k}^{\prime}\left\langle u_{k}\right\rangle: F^{\prime} \in \mathcal{P}_{f}(\omega) \text { and for all } k \in F^{\prime}, u_{k} \in \bigcup_{i=0}^{\min F^{\prime}} H_{k, i}\right\} .
$$

Let $F \in \mathcal{P}_{f}(\omega)$ and for each $n \in F$, let $u_{n} \in H$. Let $F^{\prime}=m+F$ and for $n \in F$, let $u_{m+n}^{\prime}=h_{u_{n}}\left(s_{n}\right)=u_{n} a^{n}$. Then $\min F^{\prime} \geq m$. We claim that for each $k \in F^{\prime}$, $u_{k}^{\prime} \in \bigcup_{i=0}^{\min F^{\prime}} H_{k, i}$. To see this, let $k \in F^{\prime}$ and let $n=k-m$, so that $u_{k}^{\prime}=h_{u_{n}}\left(s_{n}\right)$. Pick $i \in\{0,1, \ldots, m\}$ such that $u_{n} \in S\left(\begin{array}{c}m \\ i\end{array}\right)$. Then $u_{k}^{\prime}=u_{n} a^{n} \in H_{k, i}$. To 
complete the proof we show that for $n \in F, w_{m+n}^{\prime}\left\langle u_{m+n}^{\prime}\right\rangle=w_{n}\left\langle u_{n}\right\rangle$. Note that $h_{u_{n}} \circ h_{s_{n}}=h_{u_{m+n}^{\prime}}$. Thus

$$
w_{n}\left\langle u_{n}\right\rangle=h_{u_{n}}\left(w_{n}\right)=h_{u_{n}}\left(h_{s_{n}}\left(w_{m+n}^{\prime}\right)\right)=h_{u_{m+n}^{\prime}}\left(w_{m+n}^{\prime}\right)=w_{m+n}^{\prime}\left\langle u_{m+n}^{\prime}\right\rangle .
$$

The following corollary is then immediate.

Corollary 3.5. Assume that the alphabet $A$ is finite, that $D$ is a finite group, and that $\left\langle T_{f}\right\rangle_{f \in D}$ is an action of $D$ on $A$. Let $m, k \in \omega$ with $m>k$ and let $S_{k}$ and $S_{m}$ be finitely colored. There exists a sequence $\left\langle w_{t}\right\rangle_{t=0}^{\infty}$ in $S_{m}$ such that $\left\{\prod_{t \in F} w_{t}: F \in \mathcal{P}_{f}(\omega)\right\}$ is monochrome and $\left\{\prod_{t \in F} w_{t}\left\langle u_{t}\right\rangle: F \in \mathcal{P}_{f}(\omega)\right.$ and for each $\left.n \in F, u_{n} \in S\left(\begin{array}{c}m \\ k\end{array}\right)\right\}$ is monochrome.

Corollary 3.5 can be derived from Theorem 1.3 without using the results of Section 2 as follows. Let $k, m \in \omega$ with $m>k$. Using Theorem 1.3 show that there exist idempotents $p \in \beta S_{k}$ and $q \in \beta S_{m}$ such that $h_{u}(q)=p$ for every $u \in S\left(\begin{array}{c}m \\ k\end{array}\right)$. Then derive the corollary in a fashion similar to the proof of Theorem 3.2

Based on previous experience with many Ramsey Theoretic problems we would expect that in the statement of Theorem 1.3 one could take any color class which is central in $S_{k}$. We see now that this is not the case.

Theorem 3.6. There is a central subset $M$ of $S_{1}$ such that there is no $w \in S_{2}$ with the property that $w\langle u\rangle \in M$ for every $u \in S\left(\begin{array}{l}2 \\ 1\end{array}\right)$.

Proof. Recall that we are assuming that $A \neq \emptyset$, so pick $a \in A$. For each $k \in \mathbb{N}$, let $L_{k}=\left\{w \in S_{1}:\left|\left\{i: w(i)=v_{0}\right\}\right| \geq|\{i: w(i)=a\}|+k\right\}$ and let $L=\bigcap_{k=1}^{\infty} \overline{L_{k}}$. Trivially $L \neq \emptyset$. Given any $w \in S_{1}$, if $m=\ell(w)$, then for each $z \in L_{m+k}$, one has $w z \in L_{k}$. Consequently $L$ is a left ideal of $\beta S_{1}$. By [10, Corollary 2.6] pick a minimal idempotent $p \in L$. Let $M=L_{1}$. Then $M \in p$ so $M$ is central.

Now let $w \in S_{2}$ and suppose that $w\langle u\rangle \in M$ for every $u \in S\left(\begin{array}{l}2 \\ 1\end{array}\right)$. Let $u_{1}=a v_{0}$ and let $u_{2}=v_{0} a$. Let

$$
\begin{aligned}
b & =|\{i: w(i)=a\}|, \\
c & =\left|\left\{i: w(i)=v_{0}\right\}\right|, \text { and } \\
d & =\left|\left\{i: w(i)=v_{1}\right\}\right| .
\end{aligned}
$$

Then $d=\left|\left\{i: w\left\langle u_{1}\right\rangle(i)=v_{0}\right\}\right| \geq\left|\left\{i: w\left\langle u_{1}\right\rangle(i)=a\right\}\right|+1=b+c+1$ and $c=\left|\left\{i: w\left\langle u_{2}\right\rangle(i)=v_{0}\right\}\right| \geq\left|\left\{i: w\left\langle u_{2}\right\rangle(i)=a\right\}\right|+1=b+d+1$ and so $d \geq 2 b+d+2$, a contradiction.

\section{Some Ramsey Theoretic Consequences}

In this section we present a new and simpler derivation of a known result and a new extension of a recent result of Gunderson, Leader, Prömel, and Rödl. Both deal with the notion of a first entries matrix. Given a matrix which is denoted by an upper case letter, we shall follow the custom of denoting the entries by the lower case of the same letter. 
Definition 4.1. Let $a, b \in \mathbb{N}$ and let $M$ be an $a \times b$ matrix with entries from $\mathbb{Z}$. Then $M$ satisfies the first entries condition if and only if no row of $M$ is $\overrightarrow{0}$ and there exist $c_{1}, c_{2}, \ldots, c_{b} \in \mathbb{N}$ such that, for any row $i$ of $M$, if the first nonzero entry of row $i$ occurs in column $j$, then $m_{i, j}=c_{j}$. Each $c_{j}$ is called a first entry of $M$. A first entries matrix is a matrix with entries from $\mathbb{Z}$ which satisfies the first entries condition.

First entries matrices are based on Deuber's $(m, p, c)$-sets which were used 4 to prove Rado's conjecture about sets containing solutions to all partition regular systems of homogeneous linear equations. First entries matrices provide characterizations of all image partition regular matrices. See [10, Chapter 15].

Definition 4.2. For each $j \in \mathbb{N}$ and each $w \in W$, let $\alpha_{j}(w)$ be the number of occurrences of $v_{j-1}$ in $W$.

The following lemma will be used in both of the featured results of this section. In it, no special assumptions about the alphabet $A$ are needed.

Lemma 4.3. Let $a, b \in \mathbb{N}$, let $M$ be an $a \times b$ first entries matrix which has all first entries equal, and let $C$ be a central subset of $\mathbb{N}$. Assume that $D=E=\{e\}$. For each $i \in\{1,2, \ldots, a\}$, define $f_{i}: W \rightarrow \mathbb{Z}$ by $f_{i}(w)=\sum_{j=1}^{b} m_{i, j} \alpha_{j}(w)$. Then there is a minimal idempotent $s$ of $S_{b}$ such that $\bigcap_{i=1}^{a} f_{i}^{-1}[C] \in s$.

Proof. Pick a minimal idempotent $q^{\prime}$ of $\beta \mathbb{N}$ such that $C \in q^{\prime}$. Pick $c \in \mathbb{N}$ such that all first entries of $M$ are equal to $c$. We claim that we may choose a minimal idempotent $q$ of $\beta \mathbb{N}$ such that $c q=q^{\prime}$, where the product $c q$ is computed in the semigroup $(\beta \mathbb{N}, \cdot)$. (When we refer to the semigroup $\beta \mathbb{N}$ without an operation mentioned, we are speaking of $(\beta \mathbb{N},+)$.) To see this, define $\gamma: \beta \mathbb{N} \rightarrow c \beta \mathbb{N}$ by $\gamma(p)=c p$. Then $\gamma$ is a surjective homomorphism from $\beta \mathbb{N}$ onto $c \beta \mathbb{N}$, so by 10 , Exercise 1.7.3], $\gamma[K(\beta \mathbb{N})]=K(c \beta \mathbb{N})$. Further, by [10, Lemma 6.6], $q^{\prime} \in c \beta \mathbb{N}$ and by [10, Theorem 1.65] $K(c \beta \mathbb{N})=c \beta \mathbb{N} \cap K(\beta \mathbb{N})$, so $q^{\prime} \in K(c \beta \mathbb{N})$. Pick $r \in K(\beta \mathbb{N})$ such that $\gamma(r)=q^{\prime}$ and pick a minimal left ideal $L$ of $\beta \mathbb{N}$ such that $r \in L$. Then $L \cap \gamma^{-1}\left[\left\{q^{\prime}\right\}\right]$ is a compact right topological semigroup, and so has an idempotent $q$.

Note that for each $i \in\{1,2, \ldots, a\}, f_{i}$ is a homomorphism, so its continuous extension, also denoted by $f_{i}$, from $\beta W$ to $\beta \mathbb{Z}$ is a homomorphism. Now $\alpha_{1}\left[S_{1}\right]=\omega$, so $\alpha_{1}{ }^{-1}[\{q\}]$ is a compact subsemigroup of $\beta S_{1}$ and so we may pick an idempotent $r \in \beta S_{1}$ such that $\alpha_{1}(r)=q$. Let $p_{0}$ be any minimal idempotent of $\beta W_{0}$ and by Lemma 2.7 pick an idempotent $p_{1} \in p_{0} r \beta S_{1} \cap \beta S_{1} r p_{0}$ which is minimal in $\beta S_{1}$. Since $p_{1} \leq p_{0}$, we may pick a sequence $\left\langle p_{n}\right\rangle_{n=0}^{\infty}$ as guaranteed by Theorem 2.12 ,

Now $p_{1}=p_{0} r x$ for some $x \in \beta S_{1}$, so $\alpha_{1}\left(p_{1}\right)=\alpha_{1}\left(p_{0}\right)+\alpha_{1}(r)+\alpha_{1}(x)=$ $0+\alpha_{1}(r)+\alpha_{1}(x)$ and thus $\alpha_{1}\left(p_{1}\right) \leq_{R} \alpha_{1}(r)=q$. Similarly $\alpha_{1}(p) \leq_{L} q$. Since $q$ is minimal in $\beta \mathbb{N}$, we have $\alpha_{1}(p)=q$.

Next we observe that for any $j \in\{1,2, \ldots, b\}, \alpha_{j}\left(p_{j}\right)=q$. To see this, pick $d \in A$ and define $u \in S\left(\begin{array}{l}j \\ 1\end{array}\right)$ by agreeing that for $t \in\{0,1, \ldots, j-1\}$,

$$
u(t)=\left\{\begin{array}{cl}
d & \text { if } t \neq j-1 \\
v_{0} & \text { if } t=j-1
\end{array}\right.
$$

Then $\alpha_{j}=\alpha_{1} \circ h_{u}$ on $S_{j}$ and $h_{u}\left(p_{j}\right)=p_{1}$ by Lemma 2.14, so $\alpha_{j}\left(p_{j}\right)=\alpha_{1}\left(h_{u}\left(p_{j}\right)\right)=$ $\alpha_{1}\left(p_{1}\right)=q$. 
Now we claim that for each $i \in\{1,2, \ldots, a\}, f_{i}\left(p_{b}\right)=q^{\prime}$. This will complete the proof because we may take $s=p_{b}$. Let $i \in\{1,2, \ldots, a\}$ and let $j$ be the column with the first nonzero entry of row $i$ of $M$. Then for $w \in W, f_{i}(w)=$ $c \alpha_{j}(w)+\sum_{t=j+1}^{b} m_{i, t} \alpha_{t}(w)$. For $w \in S_{j}$ and $t>j, \alpha_{t}(w)=0$, and so on $S_{j}$, $f_{i}(w)=c \alpha_{j}(w)$ and consequently $f_{i}\left(p_{j}\right)=c \alpha_{j}\left(p_{j}\right)=c q=q^{\prime}$. Since $p_{b} \leq p_{j}$ we have that $f_{i}\left(p_{b}\right) \leq f_{i}\left(p_{j}\right)=q^{\prime}$, and so, since $q^{\prime}$ is minimal, $f_{i}\left(p_{b}\right)=q^{\prime}$.

Definition 4.4. If $M$ is an $a \times b$ matrix and $\vec{x} \in \mathbb{Z}^{b}$, then $\eta(M \vec{x})$ is the set of entries of $M \vec{x}$.

The following result is not new. (See for example [10, Theorem 15.5].) The proof here is shorter (given the earlier development).

Theorem 4.5. Let $a, b \in \mathbb{N}$ and let $M$ be an $a \times b$ first entries matrix. Let $C$ be $a$ central subset of $\mathbb{N}$. Then there exists $\vec{x} \in \mathbb{N}^{b}$ such that $\eta(M \vec{x}) \subseteq C$.

Proof. Let $c_{1}, c_{2}, \ldots, c_{b}$ be the first entries of $M$, let $c=\prod_{j=1}^{b} c_{j}$, and for $j \in\{1,2$, $\ldots, b\}$, let $d_{j}=\frac{c}{c_{j}}$. Define the $a \times b$ matrix $N$ by $n_{i, j}=d_{j} m_{i, j}$ for $i \in\{1,2$, $\ldots, a\}$ and $j \in\{1,2, \ldots, b\}$. Then $N$ is a first entries matrix with all first entries equal to $c$. Let $D=E=\{e\}$. For $i \in\{1,2, \ldots, a\}$ define $f_{i}: W \rightarrow \mathbb{Z}$ by $f_{i}(w)=\sum_{j=1}^{b} n_{i, j} \alpha_{j}(w)$. By Lemma 4.3 pick a minimal idempotent $s$ of $\beta S_{b}$ such that $\bigcap_{i=1}^{a} f_{i}{ }^{-1}[C] \in s$. Pick $w \in S_{b} \cap \bigcap_{i=1}^{a} f_{i}^{-1}[C]$. For $j \in\{1,2, \ldots, b\}$ let $x_{j}=d_{j} \alpha_{j}(w)$. Then each $x_{j} \in \mathbb{N}$, and for $i \in\{1,2, \ldots, a\}, \sum_{j=1}^{b} m_{i, j} x_{j}=$ $\sum_{j=1}^{b} d_{j} m_{i, j} \alpha_{j}(w)=\sum_{j=1}^{b} n_{i, j} \alpha_{j}(w)=f_{i}(w) \in C$.

Recently Gunderson, Leader, Prömel, and Rödl proved the following theorem. (By a $K_{k}$ we mean a complete graph on $k$ vertices. A set is independent with respect to a graph if there are no edges between members of the set.)

Theorem 4.6. Let $a, b, k \in \mathbb{N}$ and let $M$ be an $a \times b$ first entries matrix. Then there exist $c, d \in \mathbb{N}$ and $a c \times d$ first entries matrix $P$ such that all first entries of $P$ are equal, and whenever $\vec{x} \in \mathbb{N}^{d}$ and $G$ is a $K_{k}$-free graph on $\eta(P \vec{x})$, there exists $\vec{y} \in \mathbb{N}^{b}$ such that $\eta(M \vec{y})$ is an independent subset of $\eta(P \vec{x})$.

Proof. [7].

The following corollary is an immediate consequence of Theorem 4.6 .

Corollary 4.7. Let $a, b, k \in \mathbb{N}$ and let $M$ be an $a \times b$ first entries matrix. Let $G$ be a $K_{k}$-free graph on $\mathbb{N}$. Then there exists $\vec{y} \in \mathbb{N}^{b}$ such that $\eta(M \vec{y})$ is independent.

Now we establish the following simultaneous extension of Theorem 4.5 and Corollary 4.7.

Theorem 4.8. Let $a, b, k \in \mathbb{N}$, let $M$ be an $a \times b$ first entries matrix, and let $G$ be a $K_{k}$-free graph on $\mathbb{N}$. There exists a sequence $\left\langle\overrightarrow{x_{n}}\right\rangle_{n=1}^{\infty}$ in $\mathbb{N}^{b}$ such that for every $F \in \mathcal{P}_{f}(\mathbb{N}), \eta\left(M\left(\sum_{n \in F} \overrightarrow{x_{n}}\right)\right)$ is an independent subset of $C$.

Proof. By Theorem 4.6 pick some $c, d \in \mathbb{N}$ and a $c \times d$ first entries matrix $P$ with all first entries equal such that for each $\vec{z} \in \mathbb{N}^{d}$, there exists $\vec{y} \in \mathbb{N}^{b}$ such that $\eta(M \vec{y})$ is an independent subset of $\eta(P \vec{z})$. Let $D=E=\{e\}$. For $i \in\{1,2, \ldots, c\}$ define $f_{i}: W \rightarrow \mathbb{Z}$ by $f_{i}(w)=\sum_{j=1}^{d} p_{i, j} \alpha_{j}(w)$. By Lemma 4.3 pick a minimal idempotent $s$ of $\beta S_{d}$ such that $\bigcap_{i=1}^{c} f_{i}^{-1}[C] \in s$. 
For each $w \in S_{d}$, let $\overrightarrow{z_{w}}=\left(\begin{array}{c}\alpha_{1}(w) \\ \vdots \\ \alpha_{d}(w)\end{array}\right)$, pick $\overrightarrow{y_{w}} \in \mathbb{N}^{b}$ such that $\eta\left(M \overrightarrow{y_{w}}\right)$ is an independent subset of $\eta\left(P \overrightarrow{z_{w}}\right)$, and choose $\gamma_{w}:\{1,2, \ldots, a\} \rightarrow\{1,2, \ldots, c\}$ such that for each $i \in\{1,2, \ldots, a\}, \sum_{j=1}^{b} m_{i, j} y_{w, j}=\sum_{j=1}^{d} p_{\gamma_{w}(i), j} \alpha_{j}(w)$.

For each $\mu:\{1,2, \ldots, a\} \rightarrow\{1,2, \ldots, c\}$, let $H_{\mu}=\left\{w \in S_{d}: \gamma_{w}=\mu\right\}$ and pick $\mu:\{1,2, \ldots, a\} \rightarrow\{1,2, \ldots, c\}$ such that $H_{\mu} \in s$. By [10, Theorem 5.8] choose a sequence $\left\langle w_{n}\right\rangle_{n=1}^{\infty}$ in $S_{d}$ such that $F P\left(\left\langle w_{n}\right\rangle_{n=1}^{\infty}\right) \subseteq H_{\mu} \cap \bigcap_{i=1}^{c} f_{i}{ }^{-1}[C]$, where $F P\left(\left\langle w_{n}\right\rangle_{n=1}^{\infty}\right)=\left\{\prod_{n \in F} w_{n}: F \in \mathcal{P}_{f}(\mathbb{N})\right\}$ and $\prod_{n \in F} w_{n}$ is computed in increasing order of indices.

For each $n \in \mathbb{N}$, let $\overrightarrow{x_{n}}=y_{w_{n}}$. To complete the proof, let $F \in \mathcal{P}_{f}(\mathbb{N})$ and let $u=\prod_{n \in F} w_{n}$. It suffices to show that $M\left(\sum_{n \in F} \overrightarrow{x_{n}}\right)=M \overrightarrow{y_{u}}$. To this end, let $i \in\{1,2, \ldots, a\}$. The entry in row $i$ of $M\left(\sum_{n \in F} \overrightarrow{x_{n}}\right)$ is

$$
\begin{aligned}
\sum_{j=1}^{b} m_{i, j}\left(\sum_{n \in F} y_{w_{n}, j}\right) & =\sum_{n \in F} \sum_{j=1}^{b} m_{i, j} y_{w_{n}, j} \\
& =\sum_{n \in F} \sum_{j=1}^{d} p_{\mu(i), j} \alpha_{j}\left(w_{n}\right) \\
& =\sum_{j=1}^{d} p_{\mu(i), j}\left(\sum_{n \in F} \alpha_{j}\left(w_{n}\right)\right) \\
& =\sum_{j=1}^{d} p_{\mu(i), j} \alpha_{j}(u) \\
& =\sum_{j=1}^{b} m_{i, j} y_{u, j}
\end{aligned}
$$

We remark that Theorem 4.8 can be proved without using Theorem 2.12, using instead methods such as those in the proof of [11, Theorem 3.16].

\section{SOME DERIVATIONS FROM KNOWN RESUlTS}

In this section we show that the commonly quoted version of the GrahamRothschild Parameter Sets Theorem in which $D=\{e\}$ implies the full version as stated in Theorem 1.3. In fact it implies a strengthening of that full version, because in Theorem 5.1 it is not required that $D$ be a group, or even a semigroup. We also present a derivation of Corollary 3.3 for the case in which $A$ is finite and $D=\{e\}$ from [2, Theorem 10].

The following theorem may be known but is certainly not well known, even though its proof is very simple. (Before obtaining the proof we inquired of several experts whether such a derivation was possible, and none of them knew.)

Theorem 5.1. Assume the Graham Rothschild Theorem as stated in Theorem 1.3 for the case in which $D=\{e\}$. Let $\Gamma=\left(A, D,\{e\},\left\langle T_{f}\right\rangle_{f \in D}\right)$ be a parameter system for which $A$ and $D$ are finite and $T_{e}$ is the identity. Let $m, k \in \omega$ with $k<m$. Then, whenever $S_{k}(\Gamma)$ is finitely colored, there exists $w \in S_{m}(\Gamma)$ such that $\left\{w\langle u\rangle: u \in S\left(\begin{array}{c}m \\ k\end{array}\right)(\Gamma)\right\}$ is monochrome.

Proof. Let $n=|D|$ and let $D=\left\{g_{0}, g_{1}, \ldots, g_{n-1}\right\}$, with $g_{0}=e$. We define $\theta$ : $A \cup(D \times V) \rightarrow A \cup(D \times V)$ by $\theta(a)=a$ if $a \in A$ and $\theta\left(\left(g_{i}, \nu_{j}\right)\right)=\left(e, \nu_{n j+i}\right)$. As usual, let $W$ denote the semigroup of words over $A \cup(D \times V)$. Then $\theta$ extends to a homomorphism from $W$ to $W$, which we shall also denote by $\theta$. We observe that $\theta$ is a bijection from $W$ onto $W^{\prime}$, the semigroup of words over $A \cup(\{e\} \times V)$. Let $\Gamma^{\prime}$ denote the parameter system $\left(A,\{e\},\{e\},\left\langle T_{e}\right\rangle\right)$. 
We define $\lambda: D \rightarrow\{0,1, \ldots, n-1\}$ by $\lambda\left(g_{i}\right)=i$.

Since $W^{\prime} \subseteq W, h_{w}: W \rightarrow W$ is defined for every $w \in W^{\prime}$. Further, if $w \in W^{\prime}$, then $h_{w}\left[W^{\prime}\right] \subseteq W^{\prime}$. We shall show that, for every $u \in S\left(\begin{array}{c}m \\ k\end{array}\right)(\Gamma)$, there exists $u^{\prime} \in S\left(\begin{array}{c}n m \\ n k\end{array}\right)\left(\Gamma^{\prime}\right)$ such that $\theta^{-1} \circ h_{u^{\prime}} \circ \theta=h_{u}$. To this end, let $u \in S\left(\begin{array}{c}m \\ k\end{array}\right)(\Gamma)$ be given and let $t \in\{0,1, \ldots, m-1\}$. If $u(t) \in A$, we let $u^{\prime}(n t+i)=T_{g_{i}}(u(t))$ for each $i \in\{0,1, \ldots, n-1\}$. If $u(t)=\left(f, \nu_{j}\right) \in D \times V$, we put $u^{\prime}(n t+i)=\left(e, \nu_{n j+\lambda\left(g_{i} f\right)}\right)$ for each $i \in\{0,1, \ldots, n-1\}$.

We claim that $\theta^{-1} \circ h_{u^{\prime}} \circ \theta=h_{u}$. To this end, let $x \in A \cup(D \times V)$. If $x \in A$, then $\theta^{-1} \circ h_{u^{\prime}} \circ \theta(x)=x=h_{u}(x)$, so assume that $x=\left(g_{i}, \nu_{j}\right)$ for some $i \in\{0,1, \ldots, n-1\}$ and some $j \in \omega$. If $j \geq m$, then $h_{u}(x)=x$ and $\theta(x)=\left(e, \nu_{n j+i}\right)$, where $n j+i \geq n m$ so that $\theta^{-1}\left(h_{u^{\prime}}\left(e, \nu_{n j+i}\right)\right)=\theta^{-1}\left(e, \nu_{n j+i}\right)=x$. So assume that $j<m$ and assume first that $u(j) \in A$. Then $h_{u}(x)=T_{g_{i}}(u(j))$. Also $u^{\prime}(n j+i)=T_{g_{i}}(u(j))$, so $h_{u^{\prime}}(\theta(x))=T_{e}\left(T_{g_{i}}(u(j))\right)=T_{g_{i}}(u(j))$ and thus $\theta^{-1} \circ h_{u^{\prime}} \circ \theta(x)=T_{g_{i}}(u(j))$. Finally assume that $j<m$ and $u(j)=\left(f, \nu_{t}\right)$ for some $f \in D$ and some $t \in \omega$. Then $h_{u}(x)=\left(g_{i} f, \nu_{t}\right)$. Also $\theta(x)=\left(e, \nu_{n j+i}\right)$ and $u^{\prime}(n j+i)=\left(e, \nu_{n t+\lambda\left(g_{i} f\right)}\right)$, so $h_{u^{\prime}}(\theta(x))=\left(e, \nu_{n t+\lambda\left(g_{i} f\right)}\right)$. Also $\theta\left(g_{i} f, \nu_{t}\right)=\left(e, \nu_{n t+\lambda\left(g_{i} f\right)}\right)$, so $\theta^{-1} \circ h_{u^{\prime}} \circ \theta(x)=$ $\left(g_{i} f, \nu_{t}\right)$.

We must check that $u^{\prime} \in S\left(\begin{array}{c}n m \\ n k\end{array}\right)\left(\Gamma^{\prime}\right)$. Clearly, $u^{\prime}$ has length $n m$. Since $\left(e, \nu_{j}\right)$ occurs in $u$ if and only if $j \in\{0,1, \ldots, k-1\}$ and since $\lambda\left(g_{i} e\right)=i,\left(e, \nu_{n j+i}\right)$ occurs in $u^{\prime}$ if and only if $j \in\{0,1, \ldots, k-1\}$ and $i \in\{0,1, \ldots, n-1\}$. So $\left(e, \nu_{s}\right)$ occurs in $u^{\prime}$ if and only if $s \in\{0,1, \ldots, n k-1\}$.

Finally, let $j \in\{0,1, \ldots, k-1\}$ and let $i \in\{0,1, \ldots, n-1\}$. If $t$ is the first index for which $\left(e, \nu_{j}\right)$ occurs in $u$, then $n t+i$ is the first index for which $\left(e, \nu_{n j+i}\right)$ occurs in $u^{\prime}$. This establishes that $u^{\prime} \in S\left(\begin{array}{c}n m \\ n k\end{array}\right)\left(\Gamma^{\prime}\right)$.

Now it is easy to verify that $\theta^{-1}\left[S_{n k}\left(\Gamma^{\prime}\right)\right] \subseteq S_{k}(\Gamma)$ and $\theta^{-1}\left[S_{n m}\left(\Gamma^{\prime}\right)\right] \subseteq S_{m}(\Gamma)$. Let $r \in \mathbb{N}$ and let $\varphi: S_{k}(\Gamma) \rightarrow\{1,2, \ldots, r\}$ be a finite coloring of $S_{k}(\Gamma)$. Then $\varphi \circ \theta^{-1}$ is a finite coloring of $S_{n k}\left(\Gamma^{\prime}\right)$, so by Theorem 1.3 pick $w^{\prime} \in S_{n m}\left(\Gamma^{\prime}\right)$ and $i \in\{1,2, \ldots, r\}$ such that $\varphi \circ \theta^{-1}\left(h_{s}\left(w^{\prime}\right)\right)=i$ for every $s \in S\left(\begin{array}{c}n m \\ n k\end{array}\right)\left(\Gamma^{\prime}\right)$. Let $w=\theta^{-1}\left(w^{\prime}\right)$. Then $w \in S_{m}(\Gamma)$. To see that $w$ is as required, let $u \in S\left(\begin{array}{c}m \\ k\end{array}\right)$. Then

$$
\varphi(w\langle u\rangle)=\varphi\left(h_{u}(w)\right)=\varphi\left(\theta^{-1} \circ h_{u^{\prime}} \circ \theta(w)\right)=\varphi \circ \theta^{-1}\left(h_{u^{\prime}}\left(w^{\prime}\right)\right)=i .
$$

We now introduce some notation adapted from [2]. Let

$$
\mathcal{S}=\left\{\left\langle s_{n}\right\rangle_{n=0}^{\infty}: \text { for all } n \in \omega, s_{n} \text { is an } n \text {-variable word over } A\right\} .
$$

(This is, what is denoted in [2] by $\mathcal{S}^{+}(A, \vec{e})$, where $e(n)=n$ for all $n \in \omega$.)

Given $\vec{s}, \vec{t} \in \mathcal{S}, \vec{s} \leq \vec{t}$ if and only if there exists an increasing sequence $\left\langle H_{n}\right\rangle_{n=0}^{\infty}$ in $\mathcal{P}_{f}(\omega)$ (meaning that $\max H_{n}<\min H_{n+1}$ for each $n$ ), and for each $n \in \omega$ and each $k \in H_{n}$ there exists $u_{k} \in \bigcup_{l=0}^{n} S\left(\begin{array}{l}k \\ l\end{array}\right)$ such that $s_{n}=\prod_{k \in H_{n}} t_{k}\left\langle u_{k}\right\rangle$. (Since 
$s_{n} \in S_{n}$, one has that for some $k \in H_{n}, u_{k} \in S\left(\begin{array}{l}k \\ n\end{array}\right)$.) Note that $\leq$ is transitive.

(This is the relation that is denoted in $[2]$ by $\leq^{+}$.)

Give $\mathcal{S}$ the topology with basis $\{B(n, \vec{t}): n \in \omega$ and $\vec{t} \in \mathcal{S}\}$, where

$$
B(n, \vec{t})=\left\{\vec{s} \in \mathcal{S}: \vec{s} \leq \vec{t} \text { and for all } i \in\{0,1, \ldots, n-1\}, s_{i}=t_{i}\right\} .
$$

Note that if $\vec{s} \in B(n, \vec{t})$, then $B(n, \vec{s}) \subseteq B(n, \vec{t})$.

Theorem 5.2. Let $X$ be open in $\mathcal{S}$, let $\vec{s} \in \mathcal{S}$, and let $n \in \omega$. Then there exists $\vec{t} \in B(n, \vec{s})$ such that either $B(n, \vec{t}) \subseteq X$ or $B(n, \vec{t}) \cap X=\emptyset$.

Proof. This is an immediate consequence of [2, Theorem 10] because open sets are Baire.

Lemma 5.3. Let $n \in \omega$, let $k \in \mathbb{N}$, let $\varphi: S_{n} \rightarrow\{1,2, \ldots, k\}$, and let $\vec{s} \in \mathcal{S}$. There exists $\vec{t} \in B(n, \vec{s})$ such that $\varphi$ is constant on $\left\{r_{n}: \vec{r} \in B(n, \vec{t})\right\}$.

Proof. We proceed by induction on $k$, the case $k=1$ being trivial. Let $k \in \mathbb{N}$ and assume the lemma is true for $k$. Let $\varphi: S_{n} \rightarrow\{1,2, \ldots, k+1\}$ and let $X=\left\{\vec{t} \in \mathcal{S}: \varphi\left(t_{n}\right)=k+1\right\}$. If $\vec{t} \in X$, then $B(n+1, \vec{t}) \subseteq X$, so $X$ is open. Let $\vec{s} \in \mathcal{S}$ and pick $\vec{t} \in B(n, \vec{s})$ such that either $B(n, \vec{t}) \subseteq X$ or $B(n, \vec{t}) \cap X=\emptyset$.

If $B(n, \vec{t}) \subseteq X$, then $\varphi$ is constantly equal to $k+1$ on $\left\{r_{n}: \vec{r} \in B(n, \vec{t})\right\}$, so assume that $B(n, \vec{t}) \cap X=\emptyset$. Define $\tau: S_{n} \rightarrow\{1,2, \ldots, k\}$ by

$$
\tau(w)=\left\{\begin{array}{cl}
\varphi(w) & \text { if } \varphi(w) \leq k \\
1 & \text { if } \varphi(w)=k+1 .
\end{array}\right.
$$

Pick $\vec{u} \in B(n, \vec{t})$ such that $\tau$ is constant on $\left\{r_{n}: \vec{r} \in B(n, \vec{u})\right\}$. Then $\vec{u} \in B(n, \vec{s})$. We claim that $\varphi$ is constant on $\left\{r_{n}: \vec{r} \in B(n, \vec{u})\right\}$. Indeed, given $\vec{r} \in B(n, \vec{u})$ one has $\vec{r} \in B(n, \vec{t})$, so $\varphi\left(r_{n}\right) \neq k+1$ and thus $\varphi\left(r_{n}\right)=\tau\left(r_{n}\right)$.

Theorem 5.4. Assume that $A$ is finite, $D=\{e\}$, and, for each $n \in \omega, S_{n}$ has been finitely colored. Then, there exists a sequence $\left\langle w_{n}\right\rangle_{n<\omega}$ with each $w_{n} \in S_{n}$ such that for every $m \in \omega$,

$$
S_{m} \cap\left\{\prod_{n \in F} w_{n}\left\langle u_{n}\right\rangle: F \in \mathcal{P}_{f}(\omega) \text { and for all } n \in F, u_{n} \in \bigcup_{i=0}^{\min F} S\left(\begin{array}{c}
n \\
i
\end{array}\right)\right\}
$$

is monochrome. (That is, the color of $\prod_{n \in F} w_{n}\left\langle u_{n}\right\rangle$ is determined solely by the number of variables in $\prod_{n \in F} w_{n}\left\langle u_{n}\right\rangle$.)

Proof. We may assume that $T_{e}$ is the identity, since the general case then follows easily. To see this, note that whenever $w \in S\left(\begin{array}{c}n \\ m\end{array}\right)$ and $u \in S\left(\begin{array}{c}m \\ k\end{array}\right)$, there is a $u^{\prime} \in S\left(\begin{array}{c}m \\ k\end{array}\right)$ such that $w\langle u\rangle$ is the same as $w\left\langle u^{\prime}\right\rangle$, where $w\left\langle u^{\prime}\right\rangle$ is computed after reinterpreting $T_{e}$ to be the identity on $A$.

For each $n \in \omega$, let $\varphi_{n}$ be a finite coloring of $S_{n}$. By Lemma 5.3 choose $\overrightarrow{s_{0}} \in \mathcal{S}$ such that $\varphi_{0}$ is constant on $\left\{r_{0}: \vec{r} \in B\left(0, \overrightarrow{s_{0}}\right)\right\}$.

Let $n \in \mathbb{N}$ and assume that we have chosen $\overrightarrow{s_{n-1}}$. Choose $\overrightarrow{s_{n}} \in B\left(n, \overrightarrow{s_{n-1}}\right)$ such that $\varphi_{n}$ is constant on $\left\{r_{n}: \vec{r} \in B\left(n, \overrightarrow{s_{n}}\right)\right\}$.

For $n \in \omega$, let $w_{n}=s_{n, n}$, i.e. entry $n$ of $\overrightarrow{s_{n}}$. We claim that the sequence $\left\langle w_{n}\right\rangle_{n=0}^{\infty}$ is as required. So let $m \in \omega$, let $F \in \mathcal{P}_{f}(\omega)$, for each $n \in F$, let $u_{n} \in \bigcup_{i=0}^{\min F} S\left(\begin{array}{c}n \\ i\end{array}\right)$, and assume that $\prod_{n \in F} w_{n}\left\langle u_{n}\right\rangle \in S_{m}$. 
We shall show that $\prod_{n \in F} w_{n}\left\langle u_{n}\right\rangle \in\left\{r_{m}: \vec{r} \in B\left(m, \overrightarrow{s_{m}}\right)\right\}$. To this end, let $k=\max F$. Then for each $n \in F, w_{n}=s_{n, n}=s_{n+1, n}=\ldots=s_{k, n}$. Note that $m \leq \min F$ because $\prod_{n \in F} w_{n}\left\langle u_{n}\right\rangle \in S_{m}$, so for some $n, u_{n} \in S\left(\begin{array}{c}n \\ m\end{array}\right)$. For this $n$, $u_{n} \in S\left(\begin{array}{c}n \\ i\end{array}\right)$ for some $i \leq \min F$, and so $m=i$.

For $n<m$, let $r_{n}=w_{n}=s_{k, n}$. Let $r_{m}=\prod_{n \in F} w_{n}\left\langle u_{n}\right\rangle$. Also, for $n>m$, let $r_{n}=s_{k, k+n-m}\left\langle z_{n}\right\rangle$, where $z_{n}$ is any member of $S\left(\begin{array}{c}k+n-m \\ n\end{array}\right)$. Then $\vec{r} \in$ $B\left(m, \overrightarrow{s_{k}}\right) \subseteq B\left(m, \overrightarrow{s_{m}}\right)$.

\section{REFERENCES}

1. V. Bergelson, A. Blass, and N. Hindman, Partition theorems for spaces of variable words, Proc. London Math. Soc. 68 (1994), 449-476. MR1262304 (95i:05107)

2. T. Carlson, Some unifying principles in Ramsey Theory, Discrete Math. 68 (1988), 117-169. MR 0926120 (89b:04006)

3. T. Carlson, N. Hindman, and D. Strauss, Ramsey theoretic consequences of some new results about algebra in the Stone-Čech compactification, manuscript. (Currently available at http://members.aol.com/nhindman/.)

4. W. Deuber, Partitionen und lineare Gleichungssysteme, Math. Zeit. 133 (1973), 109-123. MR0325406 (48:3753)

5. H. Furstenberg, Recurrence in ergodic theory and combinatorical number theory, Princeton University Press, Princeton, 1981. MR 0603625 (82j:28010)

6. R. Graham and B. Rothschild, Ramsey's Theorem for n-parameter sets, Trans. Amer. Math. Soc. 159 (1971), 257-292. MR0284352 (44:1580)

7. D. Gunderson, I. Leader, H. Prömel, and V. Rödl, Independent Deuber sets in graphs on the natural numbers, J. Comb. Theory (Series A) 103 (2003), 305-322. MR.1996069 (2004h:05123)

8. A. Hales and R. Jewett, Regularity and positional games, Trans. Amer. Math. Soc. 106 (1963), 222-229. MR0143712 (26:1265)

9. N. Hindman, I. Leader, and D. Strauss, Infinite partition regular matrices-solutions in central sets, Trans. Amer. Math. Soc. 355 (2003), 1213-1235. MR1938754 (2003h:05187)

10. N. Hindman and D. Strauss, Algebra in the Stone-Čech compactification: Theory and applications, de Gruyter, Berlin, 1998. MR.1642231 (99j:54001)

11. N. Hindman and D. Strauss, Independent sums of arithmetic progressions in $K_{m}$-free graphs, Ars Combinatoria 70 (2004), 221-243. MR2023077 (2005a:05148)

12. P. Milnes, Compactifications of topological semigroups, J. Australian Math. Soc. 15 (1973), 488-503. MR0348030 (50:528)

13. J. Paris and L. Harrington, A mathematical incompleteness in Peano arithmetic, in Handbook of Mathematical Logic, J. Barwise, ed., North Holland, Amsterdam, 1977, 1133-1142. MR 0457132 (56:15351)

14. H. Prömel and B. Voigt, Graham-Rothschild parameter sets, in Mathematics of Ramsey Theory, J. Nešetřil and V. Rödl, eds., Springer-Verlag, Berlin, 1990, 113-149. MR1083597

Department of Mathematics, Ohio State University, Columbus, Ohio 43210

E-mail address: carlson@math.ohio-state.edu

Department of Mathematics, Howard University, Washington, DC 20059

E-mail address: nhindman@aol.com

URL: http://members .aol.com/nhindman/

Department of Pure Mathematics, University of Hull, Hull HU6 7RX, United KingDOM

E-mail address: d.strauss@maths.hull.ac.uk 